\title{
Supramolecular Energetic and Topological Study of Halogenated Aryl Carboxylic Acids
}

Patrick Teixeira Campos*, Bruno Felipe Cunha da Rosa, Henrique Saija Hilsinger, Marcéo Auler Milani, Vanessa Uecker Krüger

Laboratório de Química Orgânica Sintética, Estrutural e Computacional (LaQuiOSEC), Instituto Federal Sul-rio-grandense, IFSul , Campus Pelotas, CEP 96015-360, Pelotas, RS, Brazil

Benzoic Acid; nucleation; Cluster; intermolecular interaction; crystal packing; DFT-D; wB97X-D3.

Table of contents:

1. Table S1

2. Table S2

3. Table S3

4. Table S4

5. Table S5

6. Table S6

7. Table S8

8. Table S9

9. Table S10

10. Table S11

11. Figure S1

12. Figure $S 2$

13. Figure $S 3$

14. Figure $S 4$

15. Figure $S 5$

16. Figure $S 6$

17. Figure $S 7$

18. Figure $S 8$

19. Figure $S 9$

20. Figure $S 10$ 


\section{Figure S11}

22. Figure $S 12$

23. Figure S13

24. Figure S14

25. Figure S15

26. Figure S16

27. Figure S17

28. Figure S18

29. Figure $S 19$

30. Figure $S 20$

31. Figure S21

32. Figure S22

Table S1. Energy of M1...Mn and contact surface of M1...Mn for the supramolecular cluster of compound 1.

\begin{tabular}{|c|c|c|c|c|c|c|}
\hline Dimer & Interaction & $\mathbf{G}_{\mathrm{M} 1 \cdots \mathrm{Mn}}{ }^{\mathrm{a}}$ & $C_{M 1 \cdots M n}{ }^{b}$ & $\mathbf{N G}^{c}$ & $N C^{d}$ & $\begin{array}{c}\text { Interaction } \\
\text { type }^{9}\end{array}$ \\
\hline \multirow{2}{*}{$\mathrm{M} 1 \cdots \mathrm{M} 2$} & $\mathrm{O} 2 \ldots \mathrm{H} 1-\mathrm{O} 1$ & \multirow[t]{2}{*}{-54.78} & \multirow{2}{*}{16.8} & \multirow[t]{2}{*}{7.95} & \multirow{2}{*}{1.29} & \multirow{2}{*}{ II } \\
\hline & $\mathrm{O} 1-\mathrm{H} 1 \ldots \mathrm{O} 2$ & & & & & \\
\hline \multirow{2}{*}{ 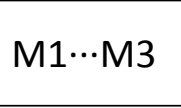 } & $\mathrm{O} 2 \ldots \mathrm{H} 5-\mathrm{C} 5$ & \multirow[t]{2}{*}{-4.04} & \multirow{2}{*}{8.21} & \multirow{2}{*}{0.59} & \multirow{2}{*}{0.63} & \multirow{2}{*}{ III } \\
\hline & F1...H5-C5 & & & & & \\
\hline $\mathrm{M} 1 \cdots \mathrm{M} 4$ & F1...H3-C3 & -2.98 & 11.21 & 0.43 & 0.86 & III \\
\hline 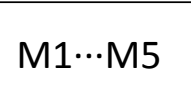 & $\mathrm{C} 4-\mathrm{H} 4 \ldots \mathrm{C} 4$ & -0.83 & 9.28 & 0.12 & 0.71 & IV \\
\hline \multirow{2}{*}{ 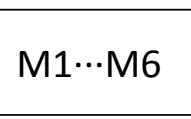 } & $\mathrm{C} 5-\mathrm{H} 5 \ldots \mathrm{O} 2$ & \multirow[t]{2}{*}{-4.04} & \multirow{2}{*}{8.21} & \multirow{2}{*}{0.59} & \multirow{2}{*}{0.63} & \multirow{2}{*}{ III } \\
\hline & C5-H5...F1 & & & & & \\
\hline \multirow{2}{*}{ 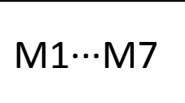 } & C6-H6...O1 & \multirow[t]{2}{*}{-2.47} & \multirow{2}{*}{18.46} & \multirow{2}{*}{0.36} & \multirow{2}{*}{1.41} & \multirow{2}{*}{ IV } \\
\hline & O1...H6-C6 & & & & & \\
\hline $\mathrm{M} 1 \cdots \mathrm{M} 8$ & Others & -2.34 & 5.44 & 0.34 & 0.42 & III \\
\hline 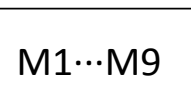 & Others & -2.77 & 10.60 & 0.40 & 0.81 & III \\
\hline 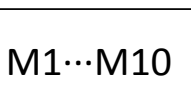 & $\pi \ldots \pi$ & -6.30 & 26.32 & 0.91 & 2.01 & IV \\
\hline 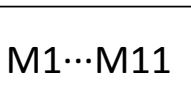 & Others & -3.08 & 11.21 & 0.45 & 0.86 & III \\
\hline 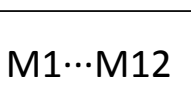 & Others & -0.73 & 9.28 & 0.11 & 0.71 & IV \\
\hline 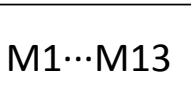 & Others & -2.75 & 10.60 & 0.40 & 0.81 & III \\
\hline M1M14 & $\pi \ldots \pi$ & -6.29 & 26.32 & 0.91 & 2.01 & IV \\
\hline M1M15 & Others & -3.06 & 11.08 & 0.44 & 0.85 & III \\
\hline
\end{tabular}

${ }^{\mathrm{a}} G_{M 1 \cdots M n}=E_{M 1 \cdots M n}-2 \times E_{M 1 .}{ }^{\mathrm{b}}$ Determined using $\operatorname{TOPOS}^{\circledR} .{ }^{12} \mathrm{c} N G_{M 1 \ldots M n}=M C N \times \frac{G_{M 1 \cdots M n}}{G_{c l u s t e r}} . \mathrm{d} N C_{M 1 \ldots M n}=M C N \times \frac{C_{M 1} \cdots M n}{C_{c l u s t e r}}$. 
Table S2. Energy of M1 $\cdots \mathrm{Mn}$ and contact surface of M1 $\cdots \mathrm{Mn}$ for the supramolecular cluster of compound 2.

\begin{tabular}{|c|c|c|c|c|c|c|c|}
\hline Dimer & Interaction & $\mathbf{G}_{\mathrm{M} 1 \cdots \mathrm{Mn}}{ }^{\mathrm{a}}$ & $C_{M 1 \cdots M n}^{b}$ & $\mathbf{N G}^{c}$ & $N C^{d}$ & $\begin{array}{c}\text { Interaction } \\
\text { type }^{9}\end{array}$ & BSSE \\
\hline \multirow{2}{*}{$\mathrm{M} 1 \cdots \mathrm{M} 2$} & $\mathrm{O} 1-\mathrm{H} 1 \ldots \mathrm{O} 2$ & \multirow{2}{*}{-16.02} & \multirow{2}{*}{13.94} & \multirow{2}{*}{3.06} & \multirow{2}{*}{0.89} & \multirow{2}{*}{$\mathbf{I}$} & \multirow{2}{*}{-3.89} \\
\hline & $\mathrm{O} 2 \ldots \mathrm{H} 1-\mathrm{O} 1$ & & & & & & \\
\hline \multirow{2}{*}{ 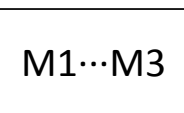 } & $\mathrm{O} 2 \ldots \mathrm{H} 5-\mathrm{C} 5$ & \multirow{2}{*}{-4.50} & \multirow[t]{2}{*}{12.53} & \multirow{2}{*}{0.86} & \multirow{2}{*}{0.80} & \multirow{2}{*}{ III } & \multirow{2}{*}{-1.54} \\
\hline & $\mathrm{Cl} 1 \ldots \mathrm{H} 5-\mathrm{C} 5$ & & & & & & \\
\hline \multirow{2}{*}{$\mathrm{M} 1 \cdots \mathrm{M} 4$} & $\mathrm{C} 3-\mathrm{H} 3 \ldots \mathrm{C} 3$ & \multirow{2}{*}{-4.38} & \multirow[t]{2}{*}{16.43} & \multirow{2}{*}{0.83} & \multirow{2}{*}{1.05} & \multirow{2}{*}{ III } & \multirow{2}{*}{-1.2} \\
\hline & $\mathrm{C} 3-\mathrm{H} 3 \ldots \mathrm{Cl} 1$ & & & & & & \\
\hline 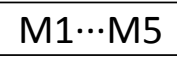 & $\mathrm{C} 4-\mathrm{H} 4 \ldots \mathrm{H} 4-\mathrm{C} 4$ & -0.21 & 5.57 & 0.04 & 0.35 & III & -0.06 \\
\hline \multirow{2}{*}{ 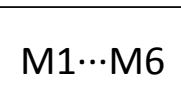 } & C5-H5...O2 & \multirow{2}{*}{-4.37} & \multirow{2}{*}{12.53} & \multirow{2}{*}{0.83} & \multirow{2}{*}{0.80} & \multirow{2}{*}{ III } & \multirow{2}{*}{-1.28} \\
\hline & $\mathrm{C} 5-\mathrm{H} 5 \ldots \mathrm{Cl} 1$ & & & & & & \\
\hline \multirow{2}{*}{$\mathrm{M} 1 \cdots \mathrm{M} 7$} & C6-H6...O1 & \multirow{2}{*}{-2.91} & \multirow{2}{*}{15.57} & \multirow{2}{*}{0.55} & \multirow{2}{*}{0.99} & \multirow{2}{*}{ III } & \multirow{2}{*}{-1.58} \\
\hline & O1...H6-C6 & & & & & & \\
\hline $\mathrm{M} 1 \cdots \mathrm{M} 8$ & Others & -2.85 & 8.60 & 0.54 & 0.55 & III & -0.87 \\
\hline 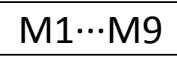 & $\pi \ldots \pi$ & -8.25 & 30.7 & 1.57 & 1.95 & II & -2.20 \\
\hline 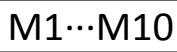 & Others & -4.36 & 16.43 & 0.83 & 1.05 & III & -1.34 \\
\hline M1M11 & Others & -2.75 & 8.60 & 0.52 & 0.55 & III & -1.39 \\
\hline 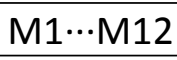 & $\pi \ldots \pi$ & -8.16 & 30.7 & 1.56 & 1.95 & II & -2.03 \\
\hline M1M13 & Others & -4.14 & 16.86 & 0.79 & 1.07 & III & -1.99 \\
\hline
\end{tabular}

Table S3. Energy of M1 ...Mn and contact surface of M1...Mn for the supramolecular cluster of compound 3.

\begin{tabular}{|c|c|c|c|c|c|c|c|}
\hline Dimer & Interaction & $\mathbf{G}_{\mathrm{M} 1 \cdots M n^{a}}$ & $C_{M 1 \cdots M n}{ }^{b}$ & $\mathbf{N G}^{c}$ & $N C^{d}$ & $\begin{array}{l}\text { Interaction } \\
\text { type }^{9}\end{array}$ & BSSE \\
\hline \multirow{2}{*}{$\mathrm{M} 1 \cdots \mathrm{M} 2$} & $\mathrm{O} 1-\mathrm{H} 1 \ldots \mathrm{O} 2$ & \multirow{2}{*}{-19.06} & \multirow{2}{*}{13.9} & \multirow{2}{*}{4.51} & \multirow{2}{*}{0.99} & \multirow{2}{*}{ I } & \multirow{2}{*}{-2.99} \\
\hline & $\mathrm{O} 2 \ldots \mathrm{H} 1-\mathrm{O} 1$ & & & & & & \\
\hline \multirow{2}{*}{ 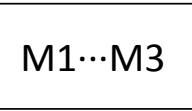 } & $\mathrm{O} 2 \ldots \mathrm{H} 5-\mathrm{C} 5$ & \multirow{2}{*}{-3.43} & \multirow[t]{2}{*}{12.35} & \multirow{2}{*}{0.81} & \multirow{2}{*}{0.89} & \multirow{2}{*}{ III } & \multirow{2}{*}{0.14} \\
\hline & $\mathrm{Br} 1 \ldots \mathrm{H} 5-\mathrm{C} 5$ & & & & & & \\
\hline \multirow{2}{*}{$\mathrm{M} 1 \cdots \mathrm{M} 4$} & $\mathrm{C} 3-\mathrm{H} 3 . . . \mathrm{C} 3$ & \multirow{2}{*}{-3.79} & \multirow[t]{2}{*}{18.85} & \multirow{2}{*}{0.90} & \multirow{2}{*}{1.35} & \multirow{2}{*}{ III } & \multirow{2}{*}{0.4} \\
\hline & $\mathrm{C} 3-\mathrm{H} 3 . . . \mathrm{Br} 1$ & & & & & & \\
\hline 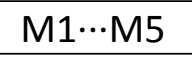 & $\mathrm{C} 4-\mathrm{H} 4 \ldots \mathrm{H} 4-\mathrm{C} 4$ & 0.27 & 2.64 & -0.06 & 0.19 & III & 0.58 \\
\hline \multirow{2}{*}{ 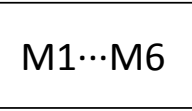 } & $\mathrm{C} 5-\mathrm{H} 5 \ldots . \mathrm{O} 2$ & \multirow{2}{*}{-3.20} & \multirow{2}{*}{12.35} & \multirow{2}{*}{0.76} & \multirow{2}{*}{0.89} & \multirow{2}{*}{ III } & \multirow{2}{*}{0.59} \\
\hline & $\mathrm{C} 5-\mathrm{H} 5 \ldots \mathrm{Br} 1$ & & & & & & \\
\hline \multirow{2}{*}{$\mathrm{M} 1 \cdots \mathrm{M} 7$} & C6-H6...O1 & \multirow{2}{*}{-2.68} & \multirow{2}{*}{18.55} & \multirow{2}{*}{0.63} & \multirow{2}{*}{1.33} & \multirow{2}{*}{ IV } & \multirow{2}{*}{-1.08} \\
\hline & O1...H6-C6 & & & & & & \\
\hline $\mathrm{M} 1 \cdots \mathrm{M} 8$ & Others & 0.048 & 0.74 & -0.01 & 0.05 & III & 1.01 \\
\hline 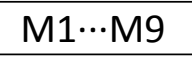 & Others & -1.97 & 10.28 & 0.47 & 0.74 & III & 0.44 \\
\hline 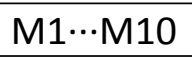 & $\pi \ldots \pi$ & -7.77 & 28 & 1.84 & 2.01 & II & 0.12 \\
\hline 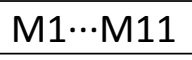 & Others & -0.14 & 1.75 & 0.03 & 0.13 & III & 0.89 \\
\hline $\mathrm{M} 1 \cdots \mathrm{M} 12$ & Others & -3.79 & 18.85 & 0.90 & 1.35 & III & 0.4 \\
\hline M1M13 & Others & -1.74 & 10.28 & 0.41 & 0.74 & III & 1.23 \\
\hline 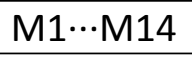 & $\pi \ldots \pi$ & -7.77 & 28 & 1.84 & 2.01 & II & -1.1 \\
\hline 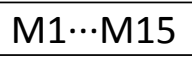 & Others & -4.14 & 18.68 & 0.98 & 1.34 & III & -1.02 \\
\hline
\end{tabular}


Table S4. Energy of M1 ...Mn and contact surface of M1...Mn for the supramolecular cluster of compound 4.

\begin{tabular}{|c|c|c|c|c|c|c|}
\hline Dimer & Interaction & $\mathrm{G}_{\mathrm{M1} \cdots \mathrm{Mn}}{ }^{\mathrm{a}}$ & $C_{M 1 \cdots M n}{ }^{b}$ & $\mathbf{N G}^{c}$ & $N C^{d}$ & $\begin{array}{c}\text { Interaction } \\
\text { type }^{9}\end{array}$ \\
\hline \multirow{2}{*}{$\mathrm{M} 1 \cdots \mathrm{M} 2$} & $\mathrm{O} 1-\mathrm{H} 1 \ldots \mathrm{O} 2$ & \multirow{2}{*}{-19.74} & \multirow{2}{*}{14.3} & \multirow{2}{*}{2.36} & \multirow{2}{*}{0.86} & \multirow{2}{*}{ I } \\
\hline & $\mathrm{O} 2 \ldots \mathrm{H} 1-\mathrm{O} 1$ & & & & & \\
\hline \multirow{2}{*}{$\mathrm{M} 1 \cdots \mathrm{M} 3$} & $\mathrm{O} 2 \ldots \mathrm{H} 4-\mathrm{C} 4$ & \multirow{2}{*}{-6.64} & \multirow[t]{2}{*}{12.24} & \multirow{2}{*}{0.80} & \multirow{2}{*}{0.73} & \multirow{2}{*}{ III } \\
\hline & $\mathrm{I1} \ldots \mathrm{H} 4-\mathrm{C} 4$ & & & & & \\
\hline \multirow{2}{*}{$\mathrm{M} 1 \cdots \mathrm{M} 4$} & $\mathrm{C} 3-\mathrm{H} 3 \ldots \mathrm{H} 6-\mathrm{C} 6$ & \multirow{2}{*}{-6.14} & \multirow[t]{2}{*}{19.09} & \multirow{2}{*}{0.74} & \multirow{2}{*}{1.14} & \multirow{2}{*}{ III } \\
\hline & $\mathrm{C} 3-\mathrm{H} 3 \ldots \mathrm{O} 1$ & & & & & \\
\hline \multirow{2}{*}{ 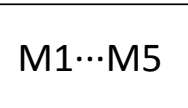 } & $\mathrm{C} 4-\mathrm{H} 4 \ldots \mathrm{O} 2$ & \multirow{2}{*}{-6.64} & \multirow{2}{*}{12.24} & \multirow{2}{*}{0.80} & \multirow{2}{*}{0.73} & \multirow{2}{*}{ III } \\
\hline & C4-H4...I1 & & & & & \\
\hline \multirow{2}{*}{$\mathrm{M} 1 \cdots \mathrm{M} 6$} & $\mathrm{C} 6-\mathrm{H} 6 \ldots \mathrm{H} 3-\mathrm{C} 3$ & \multirow{2}{*}{-5.87} & \multirow{2}{*}{19.09} & \multirow{2}{*}{0.70} & \multirow{2}{*}{1.14} & \multirow{2}{*}{ III } \\
\hline & O1...H3-C3 & & & & & \\
\hline $\mathrm{M} 1 \cdots \mathrm{M} 7$ & Others & -7.68 & 20.82 & 0.92 & 1.25 & III \\
\hline $\mathrm{M} 1 \cdots \mathrm{M} 8$ & $\pi \ldots \pi$ & -11.66 & 28.82 & 1.40 & 1.73 & II \\
\hline 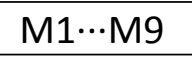 & Others & -5.69 & 9.96 & 0.68 & 0.60 & III \\
\hline 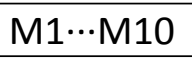 & Others & -7.68 & 20.82 & 0.92 & 1.25 & III \\
\hline M1M11 & Others & -5.35 & 4.27 & 0.64 & 0.26 & III \\
\hline $\mathrm{M} 1 \cdots \mathrm{M} 12$ & $\pi \ldots \pi$ & -11.38 & 28.82 & 1.36 & 1.73 & II \\
\hline 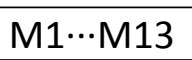 & Others & -5.69 & 9.96 & 0.68 & 0.60 & III \\
\hline
\end{tabular}

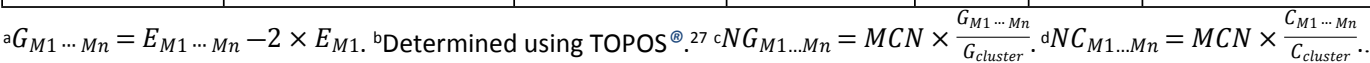

Table S5. Energy of M1 ...Mn and contact surface of M1...Mn for the supramolecular cluster of compound 5.

\begin{tabular}{|c|c|c|c|c|c|c|c|}
\hline Dimer & Interaction & $\mathrm{G}_{\mathrm{M} 1 \cdots \mathrm{Mn}}{ }^{\mathrm{a}}$ & $C_{M 1 \cdots M n}{ }^{b}$ & $\mathbf{N G}^{c}$ & $N C^{d}$ & $\begin{array}{c}\text { Interaction } \\
\text { type }^{9}\end{array}$ & BSSE \\
\hline \multirow{2}{*}{$\mathrm{M} 1 \cdots \mathrm{M} 2$} & $\mathrm{O} 1-\mathrm{H} 1 \ldots \mathrm{O} 2$ & \multirow[t]{2}{*}{-53.59} & \multirow{2}{*}{16.94} & \multirow{2}{*}{7.67} & \multirow{2}{*}{1.29} & \multirow{2}{*}{ II } & \multirow{2}{*}{-4.04} \\
\hline & O2...H101 & & & & & & \\
\hline 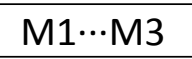 & O2...H5-C5 & -3.18 & 6.6 & 0.45 & 0.50 & III & -0.63 \\
\hline \multirow{2}{*}{$\mathrm{M} 1 \cdots \mathrm{M} 4$} & $\mathrm{C} 2-\mathrm{H} 2 \ldots \mathrm{F} 1$ & \multirow{2}{*}{-2.80} & \multirow{2}{*}{11.71} & \multirow{2}{*}{0.40} & \multirow{2}{*}{0.89} & \multirow{2}{*}{ III } & \multirow{2}{*}{-1.51} \\
\hline & F1...F1 & & & & & & \\
\hline 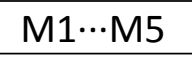 & $\mathrm{C} 4-\mathrm{H} 4 \ldots \mathrm{F} 1$ & -2.98 & 11.49 & 0.43 & 0.88 & III & -0.62 \\
\hline 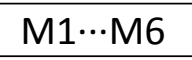 & C5-H5...O2 & -3.02 & 6.6 & 0.44 & 0.50 & III & -0.31 \\
\hline \multirow{2}{*}{$\mathrm{M} 1 \cdots \mathrm{M} 7$} & C6-H6...O1 & \multirow{2}{*}{-2.71} & \multirow{2}{*}{18.59} & \multirow{2}{*}{0.39} & \multirow{2}{*}{1.42} & \multirow{2}{*}{ IV } & \multirow{2}{*}{-1.03} \\
\hline & O1...H6-C6 & & & & & & \\
\hline $\mathrm{M} 1 \cdots \mathrm{M} 8$ & Others & -3.29 & 12 & 0.47 & 0.91 & III & -0.65 \\
\hline 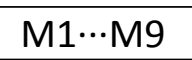 & $\pi \ldots \pi$ & -7.00 & 26.7 & 1.01 & 2.04 & II & -1.51 \\
\hline 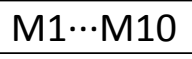 & Others & -2.08 & 8.73 & 0.30 & 0.66 & III & -0.24 \\
\hline M1M11 & Others & -2.97 & 11.49 & 0.42 & 0.88 & III & -0.63 \\
\hline $\mathrm{M} 1 \cdots \mathrm{M} 12$ & Others & -2.81 & 11.71 & 0.40 & 0.89 & III & -1.47 \\
\hline 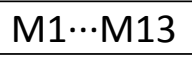 & $\pi \ldots \pi$ & -6.97 & 26.7 & 1.01 & 2.04 & II & -1.46 \\
\hline M1M14 & Others & -2.21 & 8.73 & 0.32 & 0.66 & III & -0.5 \\
\hline 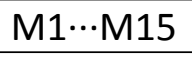 & Others & -2.22 & 4.67 & 0.32 & 0.35 & III & 0.1 \\
\hline
\end{tabular}

${ }^{\mathrm{a}} G_{M 1 \cdots M n}=E_{M 1 \cdots M n}-2 \times E_{M 1 .}{ }^{\mathrm{b}}$ Determined using $\operatorname{TOPOS}^{\circledR} .{ }^{27} \mathrm{c} N G_{M 1 \ldots M n}=M C N \times \frac{G_{M 1 \cdots M n}}{G_{\text {cluster }}} . \mathrm{d} N C_{M 1 \ldots M n}=M C N \times \frac{C_{M 1 \cdots M n}}{C_{\text {cluster }}}$. 
Table S6. Energy of M1...Mn and contact surface of M1...Mn for the supramolecular cluster of compound 6

\begin{tabular}{|c|c|c|c|c|c|c|}
\hline Dimer & Interaction & $\mathbf{G}_{M 1 \cdots M n^{a}}$ & $C_{M 1 \cdots M n}^{b}$ & NGc & $N C^{d}$ & $\begin{array}{c}\text { Interaction } \\
\text { type }^{9}\end{array}$ \\
\hline \multirow[t]{2}{*}{ 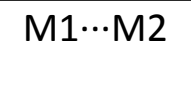 } & O1-H1...O2 & \multirow{2}{*}{-21.30} & \multirow{2}{*}{15.47} & \multirow{2}{*}{4.47} & \multirow{2}{*}{1.11} & \multirow{2}{*}{ II } \\
\hline & $\mathrm{O} 2 \ldots \mathrm{H} 1-\mathrm{O} 1$ & & & & & \\
\hline \multirow[t]{2}{*}{ 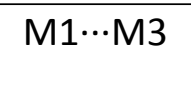 } & O2...H4-C4 & \multirow{2}{*}{-4.97} & \multirow{2}{*}{16.12} & \multirow{2}{*}{1.04} & \multirow{2}{*}{1.16} & \multirow{2}{*}{ II } \\
\hline & $\mathrm{C} 2-\mathrm{H} 2 \ldots \mathrm{Cl} 1$ & & & & & \\
\hline 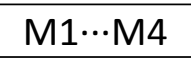 & C3-H3...O1 & -0.93 & 8.33 & 0.20 & 0.60 & III \\
\hline \multirow[t]{2}{*}{ 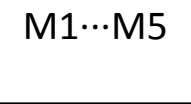 } & C4-H4...O2 & \multirow{2}{*}{-4.97} & \multirow{2}{*}{16.12} & \multirow{2}{*}{1.04} & \multirow{2}{*}{1.16} & \multirow[t]{2}{*}{ II } \\
\hline & $\mathrm{Cl} 1 \ldots \mathrm{H} 2-\mathrm{C} 2$ & & & & & \\
\hline \multirow[t]{2}{*}{ 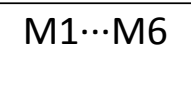 } & $\mathrm{Cl} 1-\mathrm{H} 6-\mathrm{C} 6$ & \multirow{2}{*}{-1.90} & \multirow{2}{*}{10.82} & \multirow{2}{*}{0.40} & \multirow{2}{*}{0.78} & \multirow{2}{*}{ III } \\
\hline & C6-H6...Cl1 & & & & & \\
\hline 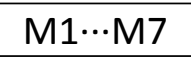 & O1...H3-C3 & -0.93 & 8.33 & 0.20 & 060 & III \\
\hline 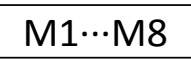 & Others & -2.33 & 5.95 & 0.49 & 0.43 & III \\
\hline 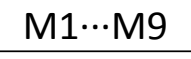 & Others & -4.40 & 14.41 & 0.92 & 1.03 & III \\
\hline 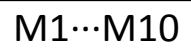 & $\pi \ldots \pi$ & -7.95 & 31.24 & 1.67 & 2.24 & II \\
\hline M1M11 & Others & -4.40 & 14.41 & 0.92 & 1.03 & III \\
\hline $\mathrm{M} 1 \cdots \mathrm{M} 12$ & Others & -0.58 & 3.62 & 0.12 & 0.26 & III \\
\hline M1M13 & Others & -3.57 & 15.55 & 0.75 & 1.12 & III \\
\hline 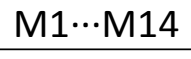 & $\pi \ldots \pi$ & -7.96 & 31.24 & 1.67 & 2.24 & II \\
\hline 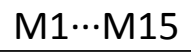 & Others & -0.58 & 3.62 & 0.12 & 0.26 & III \\
\hline
\end{tabular}

Table S7. Energy of M1...Mn and contact surface of M1...Mn for the supramolecular cluster of compound 7.

\begin{tabular}{|c|c|c|c|c|c|c|}
\hline Dimer & Interaction & $\mathbf{G}_{\mathrm{M} 1 \cdots M n^{a}}$ & $C_{M 1 \cdots M n}{ }^{b}$ & NG $^{c}$ & $N C^{d}$ & $\begin{array}{c}\text { Interaction } \\
\text { type }^{9}\end{array}$ \\
\hline \multirow{2}{*}{$\mathrm{M} 1 \cdots \mathrm{M} 2$} & $\mathrm{O} 1-\mathrm{H} 1 \ldots \mathrm{O} 2$ & \multirow{2}{*}{-16.05} & \multirow{2}{*}{16.28} & \multirow{2}{*}{3.31} & \multirow{2}{*}{1.12} & \multirow{2}{*}{ II } \\
\hline & $\mathrm{O} 2 \ldots \mathrm{H} 1-\mathrm{O} 1$ & & & & & \\
\hline $\mathrm{M} 1 \cdots \mathrm{M} 3$ & $\mathrm{O} 2 \ldots \mathrm{H} 5-\mathrm{C} 5$ & -3.85 & 8.73 & 0.79 & 0.60 & III \\
\hline 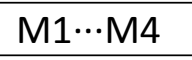 & $\mathrm{Br} 1 \ldots \mathrm{Br} 1$ & -1.67 & 5.72 & 0.35 & 0.39 & III \\
\hline 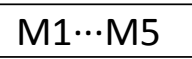 & $\mathrm{C} 4-\mathrm{H} 4 \ldots \mathrm{Br} 1$ & -3.82 & 15.37 & 0.79 & 1.06 & III \\
\hline 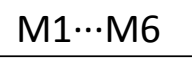 & $\mathrm{C} 5-\mathrm{H} 5 \ldots \mathrm{O} 2$ & -3.85 & 8.73 & 0.79 & 0.60 & III \\
\hline \multirow{2}{*}{$\mathrm{M} 1 \cdots \mathrm{M} 7$} & C6-H6...O1 & \multirow{2}{*}{-2.87} & \multirow{2}{*}{17.74} & \multirow{2}{*}{0.59} & \multirow{2}{*}{1.22} & \multirow{2}{*}{ IV } \\
\hline & O1...H6-C6 & & & & & \\
\hline $\mathrm{M} 1 \cdots \mathrm{M} 8$ & Others & -6.19 & 15.72 & 1.28 & 1.08 & II \\
\hline M1M9 & Others & -4.70 & 18.46 & 0.97 & 1.27 & III \\
\hline M1M10 & $\pi \ldots \pi$ & -6.80 & 27.37 & 1.40 & 1.89 & II \\
\hline M1M11 & Others & -1.67 & 5.72 & 0.35 & 0.39 & III \\
\hline 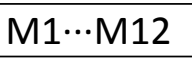 & Others & -4.70 & 18.46 & 0.97 & 1.27 & III \\
\hline M1M13 & Others & -3.82 & 15.37 & 0.79 & 1.06 & III \\
\hline M1M14 & $\pi \ldots \pi$ & -6.80 & 27.37 & 1.40 & 1.89 & II \\
\hline 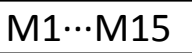 & Others & -1.11 & 1.93 & 0.23 & 0.13 & III \\
\hline
\end{tabular}


Table S8. Energy of M1 ...Mn and contact surface of M1...Mn for the supramolecular cluster of compound 8.

\begin{tabular}{|c|c|c|c|c|c|c|c|}
\hline Dimer & Interaction & $\mathrm{G}_{\mathrm{M} 1 \cdots \mathrm{Mn}^{\mathrm{a}}}$ & $C_{M 1 \cdots M n}^{b}$ & $\mathbf{N G}^{c}$ & $N C^{d}$ & $\begin{array}{l}\text { Interaction } \\
\text { type }^{9}\end{array}$ & BSSE \\
\hline \multirow[t]{2}{*}{$\mathrm{M} 1 \cdots \mathrm{M} 2$} & $\mathrm{O} 1-\mathrm{H} 1 \ldots \mathrm{O} 2$ & \multirow{2}{*}{-34.23} & \multirow{2}{*}{17.9} & \multirow{2}{*}{6.54} & \multirow{2}{*}{1.19} & \multirow{2}{*}{ II } & \multirow{2}{*}{-3.67} \\
\hline & O2...H1-O1 & & & & & & \\
\hline \multirow[t]{2}{*}{ 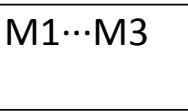 } & $\mathrm{O} 2 \ldots \mathrm{H} 2-\mathrm{C} 2$ & \multirow{2}{*}{-6.80} & \multirow[t]{2}{*}{12.79} & \multirow{2}{*}{1.30} & \multirow{2}{*}{0.85} & \multirow{2}{*}{ I } & \multirow{2}{*}{-1.1} \\
\hline & $\mathrm{C} 2-\mathrm{H} 2 \ldots \mathrm{O} 2$ & & & & & & \\
\hline 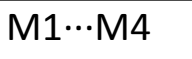 & C3-H3...O1 & -0.50 & 11.21 & 0.10 & 0.74 & IV & 0.25 \\
\hline 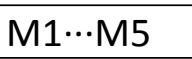 & C4-H4...I1 & -2.63 & 15.92 & 0.50 & 1.05 & IV & 0.71 \\
\hline 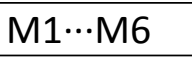 & $|1 \ldots| 1$ & -0.59 & 5.15 & 0.11 & 0.34 & III & 1.09 \\
\hline 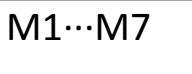 & С3-H3...O1 & -0.48 & 11.21 & 0.09 & 0.74 & IV & 0.29 \\
\hline 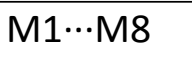 & Others & -4.34 & 13.25 & 0.83 & 0.88 & III & -0.35 \\
\hline 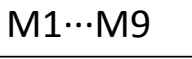 & Others & -4.06 & 20.3 & 0.78 & 1.35 & IV & 0.21 \\
\hline 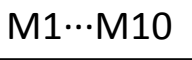 & $\pi \ldots \pi$ & -6.78 & 30.1 & 1.29 & 2.00 & II & -0.25 \\
\hline 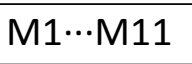 & Others & -0.59 & 5.15 & 0.11 & 0.34 & III & 1.09 \\
\hline 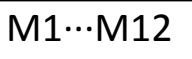 & Others & -4.08 & 20.3 & 0.78 & 1.35 & IV & 0.17 \\
\hline M1M13 & Others & -2.62 & 15.92 & 0.50 & 1.05 & IV & 0.71 \\
\hline 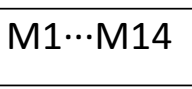 & $\pi \ldots \pi$ & -6.78 & 30.1 & 1.29 & 2.00 & II & -0.25 \\
\hline 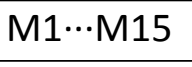 & Others & 1.19 & 1.15 & -0.23 & 0.07 & III & 1.12 \\
\hline
\end{tabular}

Table S9. Energy of M1...Mn and contact surface of M1...Mn for the supramolecular cluster of compound 9.

\begin{tabular}{|c|c|c|c|c|c|c|c|}
\hline Dimer & Interaction & $\mathrm{G}_{\mathrm{M} 1 \cdots \mathrm{Mn}}{ }^{\mathrm{a}}$ & $C_{M 1 \cdots M n}{ }^{b}$ & $N^{c}{ }^{c}$ & $N C^{d}$ & $\begin{array}{l}\text { Interaction } \\
\text { type }^{9}\end{array}$ & BSSE \\
\hline $\mathrm{M} 1 \cdots \mathrm{M} 2$ & $\mathrm{O} 2-\mathrm{H} 1 \cdots \mathrm{O} 1$ & -23.65 & 15.20 & 4.97 & 1.16 & II & -4.06 \\
\hline $\mathrm{M} 1 \cdots \mathrm{M} 3$ & $\mathrm{C} 5-\mathrm{H} 5 \cdots \mathrm{O} 2$ & -4.08 & 10.78 & 0.86 & 0.82 & III & -1.03 \\
\hline 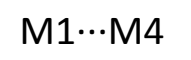 & $\mathrm{C} 3-\mathrm{H} 3 \cdots \mathrm{F} 1$ & -2.07 & 10.58 & 0.43 & 0.81 & III & -0.93 \\
\hline 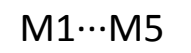 & $\mathrm{C} 3-\mathrm{H} 3 \cdots \mathrm{F} 1$ & -1.58 & 7.87 & 0.33 & 0.60 & III & -0.73 \\
\hline 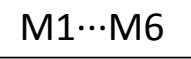 & $\mathrm{C} 5-\mathrm{H} 5 \cdots \mathrm{O} 2$ & -4.04 & 10.78 & 0.85 & 0.82 & III & -0.93 \\
\hline $\mathrm{M} 1 \cdots \mathrm{M} 7$ & $\mathrm{C} 6-\mathrm{H} 6 \cdots \mathrm{O} 1$ & -2.38 & 16.16 & 0.50 & 1.23 & IV & -1.5 \\
\hline $\mathrm{M} 1 \cdots \mathrm{M} 8$ & Others & -2.73 & 4.65 & 0.57 & 0.35 & III & -1 \\
\hline $\mathrm{M} 1 \cdots \mathrm{M} 9$ & $\pi \ldots \pi$ & -7.41 & 27.13 & 1.56 & 2.07 & II & -1.99 \\
\hline 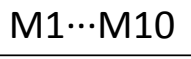 & Others & -2.93 & 12.68 & 0.62 & 0.97 & III & -0.8 \\
\hline M1M11 & Others & -2.07 & 7.87 & 0.43 & 0.60 & III & -0.93 \\
\hline $\mathrm{M} 1 \cdots \mathrm{M} 12$ & Others & -1.58 & 10.58 & 0.33 & 0.81 & III & -0.73 \\
\hline M1M13 & $\pi \ldots \pi$ & -7.39 & 27.13 & 1.55 & 2.07 & II & -1.97 \\
\hline 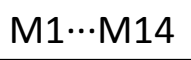 & Others & -2.96 & 12.68 & 0.62 & 0.97 & III & -1.97 \\
\hline 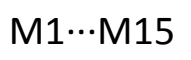 & Others & -1.73 & 9.72 & 0.36 & 0.74 & III & -0.19 \\
\hline
\end{tabular}


Table S10. Energy of M1...Mn and contact surface of M1...Mn for the supramolecular cluster of compound 10.

\begin{tabular}{|c|c|c|c|c|c|c|c|}
\hline Dimer & Interaction & $\mathrm{G}_{\mathrm{M} 1 \cdots \mathrm{Mn}}{ }^{\mathrm{a}}$ & $\mathrm{C}_{\mathrm{M} 1 \cdots \mathrm{Mn}}{ }^{\mathrm{b}}$ & $\mathbf{N G}^{c}$ & $N C^{d}$ & $\begin{array}{l}\text { Interaction } \\
\text { type }^{9}\end{array}$ & BSSE \\
\hline $\mathrm{M} 1 \cdots \mathrm{M} 2$ & $\mathrm{O} 2-\mathrm{H} 1 \cdots \mathrm{O} 1$ & -17.82 & 14.36 & 3.83 & 1.05 & II & -4.76 \\
\hline $\mathrm{M} 1 \cdots \mathrm{M} 3$ & $\mathrm{C} 5-\mathrm{H} 5 \cdots \mathrm{O} 2$ & -2.43 & 10.88 & 0.52 & 0.80 & III & -1.67 \\
\hline $\mathrm{M} 1 \cdots \mathrm{M} 4$ & $\mathrm{C} 3-\mathrm{H} 3 \cdots \mathrm{Cl} 1$ & -4.12 & 15.80 & 0.89 & 1.16 & III & -1.03 \\
\hline 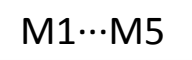 & $\mathrm{Cl} 1 \cdots \mathrm{Cl} 1$ & -0.40 & 5.35 & 0.09 & 0.39 & III & -0.19 \\
\hline $\mathrm{M} 1 \cdots \mathrm{M} 6$ & $\mathrm{C} 5-\mathrm{H} 5 \cdots \mathrm{O} 2$ & -2.91 & 10.88 & 0.63 & 0.80 & III & -0.59 \\
\hline $\mathrm{M} 1 \cdots \mathrm{M} 7$ & $\mathrm{C} 6-\mathrm{H} 6 \cdots \mathrm{O} 1$ & -4.36 & 15.33 & 0.94 & 1.12 & III & -1.5 \\
\hline $\mathrm{M} 1 \cdots \mathrm{M} 8$ & Others & -1.77 & 5.02 & 0.38 & 0.37 & III & -0.51 \\
\hline 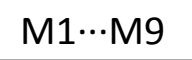 & Others & -3.89 & 16.01 & 0.84 & 1.17 & III & -0.93 \\
\hline $\mathrm{M} 1 \cdots \mathrm{M} 10$ & $\pi \ldots \pi$ & -8.38 & 29.96 & 1.80 & 2.20 & II & -2.05 \\
\hline M1M11 & Others & -2.76 & 10.42 & 0.59 & 0.74 & III & -0.97 \\
\hline $\mathrm{M} 1 \cdots \mathrm{M} 12$ & Others & -0.57 & 1.37 & 0.12 & 0.10 & III & -0.32 \\
\hline $\mathrm{M} 1 \cdots \mathrm{M} 13$ & Others & -4.26 & 16.01 & 0.92 & 1.17 & III & -1.67 \\
\hline M1M14 & $\pi \ldots \pi$ & -8.66 & 29.96 & 1.86 & 2.20 & II & -2.62 \\
\hline M1M15 & Others & -2.75 & 9.34 & 0.59 & 0.68 & III & -1.49 \\
\hline
\end{tabular}

Table S11. Energy of M1...Mn and contact surface of M1...Mn for the supramolecular cluster of compound 12.

\begin{tabular}{|c|c|c|c|c|c|c|}
\hline Dimer & Interaction & $\mathbf{G}_{\mathrm{M} 1 \cdots M n^{a}}$ & $\mathrm{C}_{\mathrm{M} 1 \cdots \mathrm{Mn}}{ }^{\mathrm{b}}$ & $\mathbf{N G}^{c}$ & $N C^{d}$ & $\begin{array}{c}\text { Interaction } \\
\text { type }^{9}\end{array}$ \\
\hline $\mathrm{M} 1 \cdots \mathrm{M} 2$ & $\mathrm{O} 2-\mathrm{H} 1 \cdots \mathrm{O} 1$ & -35.53 & 17.70 & 5.93 & 1.19 & II \\
\hline $\mathrm{M} 1 \cdots \mathrm{M} 3$ & $\mathrm{C} 5-\mathrm{H} 5 \cdots \mathrm{O} 2$ & -4.98 & 14.39 & 0.83 & 0.97 & III \\
\hline 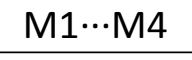 & $\mathrm{C} 3-\mathrm{H} 3 \cdots 11$ & -1.76 & 7.93 & 0.29 & 0.53 & III \\
\hline 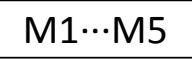 & $|1 \cdots| 1$ & -2.01 & 7.90 & 0.33 & 0.53 & III \\
\hline 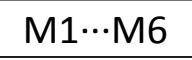 & $\mathrm{C} 5-\mathrm{H} 5 \cdots \mathrm{O} 2$ & -5.13 & 14.39 & 0.86 & 0.97 & III \\
\hline $\mathrm{M} 1 \cdots \mathrm{M} 7$ & $\mathrm{C} 6-\mathrm{H} 6 \cdots \mathrm{O} 1$ & -2.81 & 17.35 & 0.47 & 1.17 & IV \\
\hline 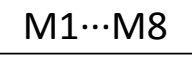 & Others & -2.55 & 10.06 & 0.43 & 0.68 & III \\
\hline 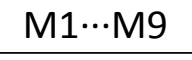 & $\pi \ldots \pi$ & -7.40 & 30.63 & 1.23 & 2.07 & II \\
\hline 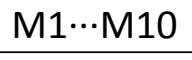 & Others & -4.30 & 17.91 & 0.72 & 1.21 & III \\
\hline 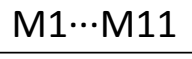 & Others & -1.96 & 7.93 & 0.33 & 0.53 & III \\
\hline 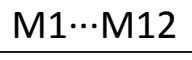 & Others & -1.80 & 7.90 & 0.30 & 0.53 & III \\
\hline $\mathrm{M} 1 \cdots \mathrm{M} 13$ & $\pi \ldots \pi$ & -7.52 & 30.63 & 1.25 & 2.07 & II \\
\hline 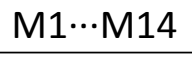 & Others & -4.28 & 17.91 & 0.72 & 1.21 & III \\
\hline 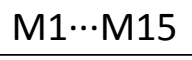 & Others & -1.90 & 5.03 & 0.32 & 0.34 & III \\
\hline
\end{tabular}

${ }^{a} G_{M 1 \cdots M n}=E_{M 1 \cdots M n}-2 \times E_{M 1 .}{ }^{b}$ Determined using $\operatorname{TOPOS}^{\circledR} .27 c N G_{M 1 \ldots M n}=M C N \times \frac{G_{M 1} \cdots M n}{G_{c l u s t e r}} . d N C_{M 1 \ldots M n}=M C N \times \frac{C_{M 1} \cdots M n}{C_{\text {cluster }}}$ 


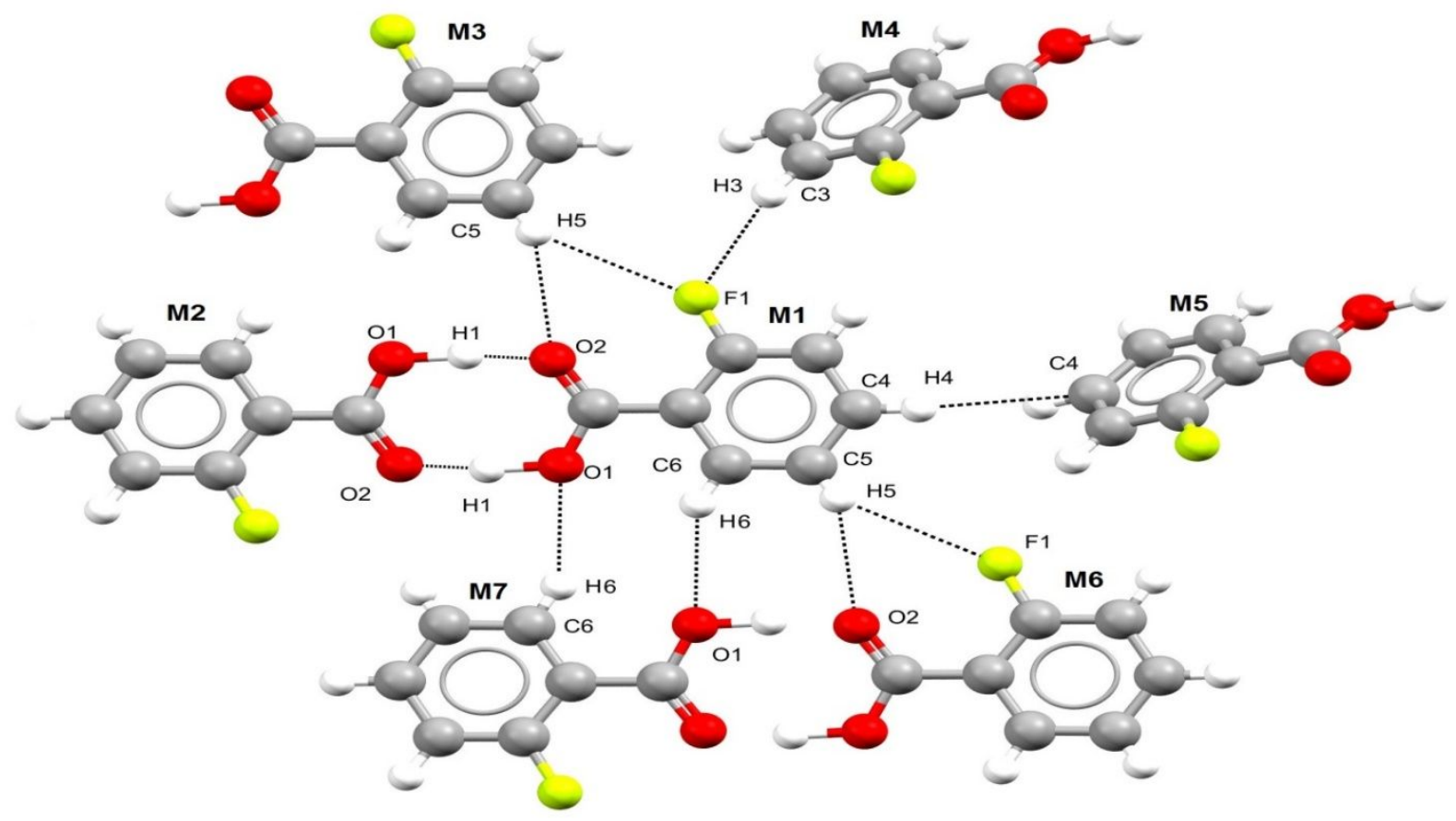

Figure S1. Supramolecular cluster showing central molecule with neighboring molecules in the same plane for compound $\mathbf{1}$.

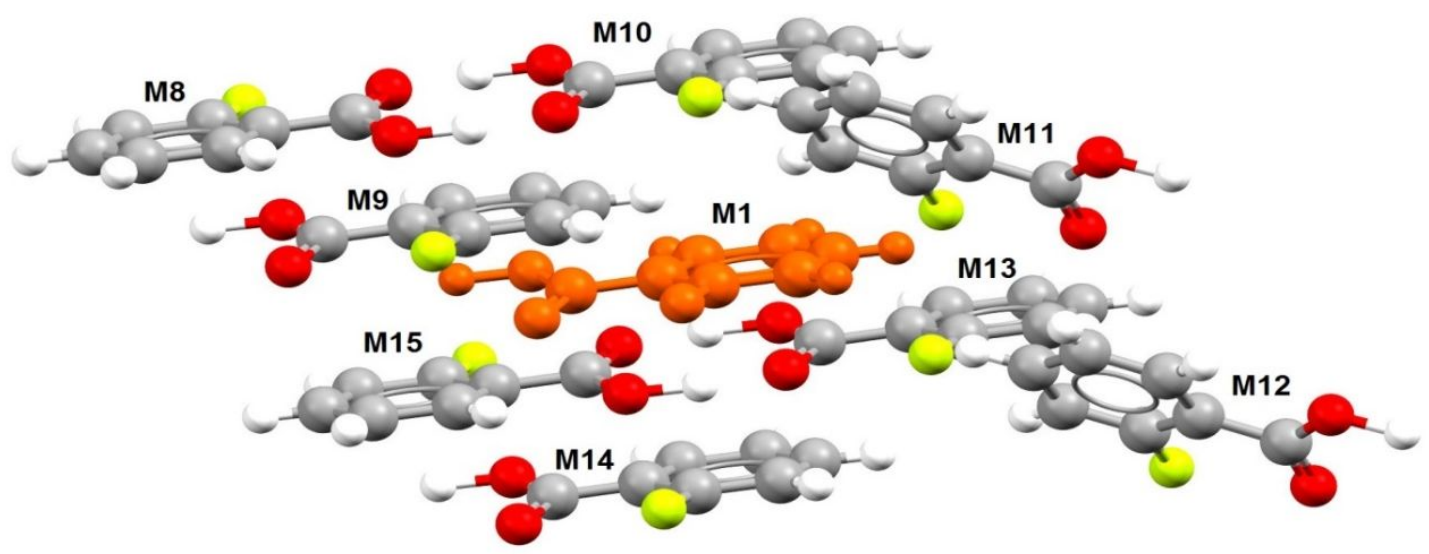

Figure S2. Supramolecular cluster showing central molecule with neighboring molecules in upper and lower layers for compound $\mathbf{1 .}$ 


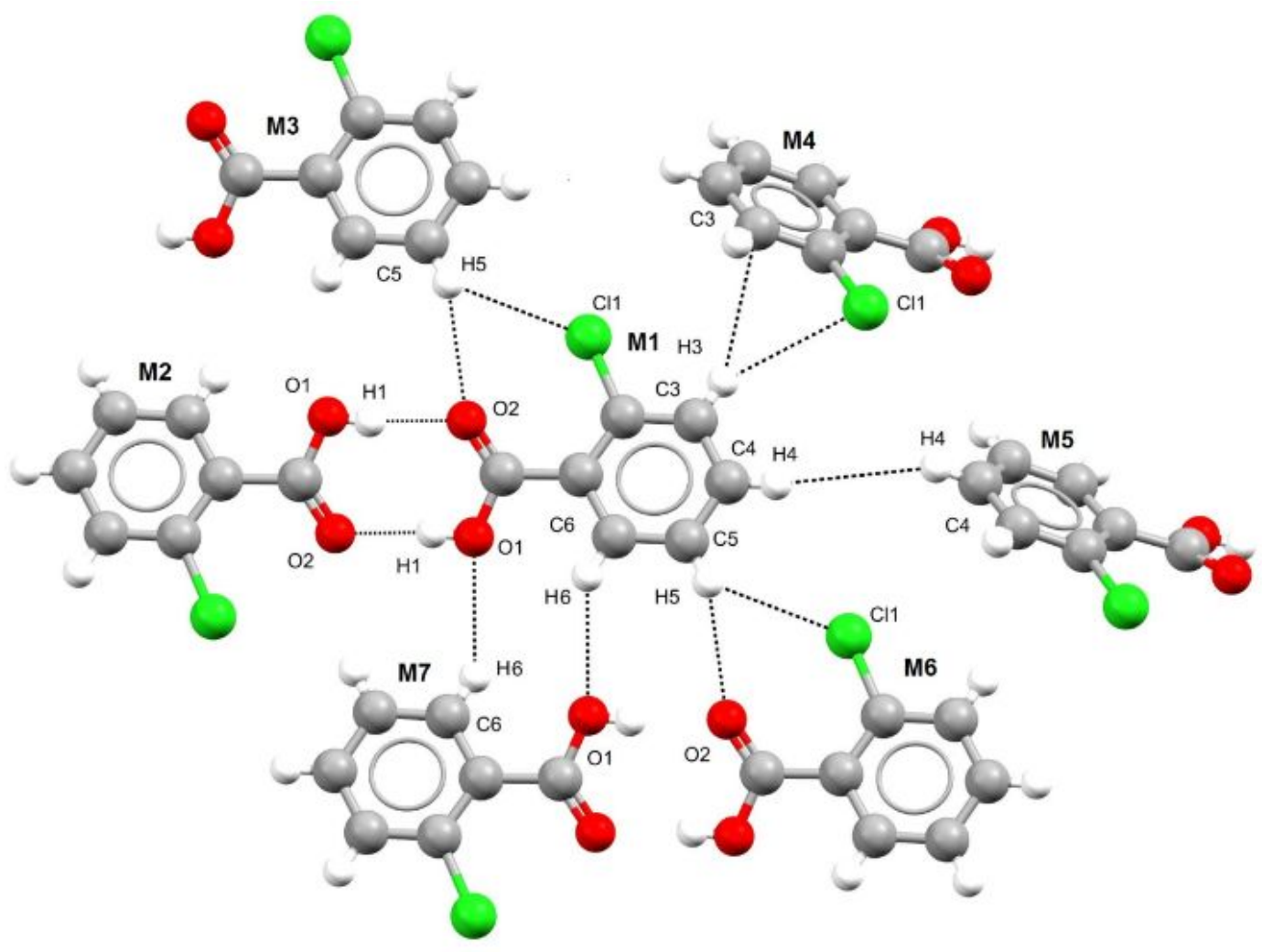

Figure S3. Supramolecular cluster showing central molecule with neighboring molecules in the same plane for compound 2.

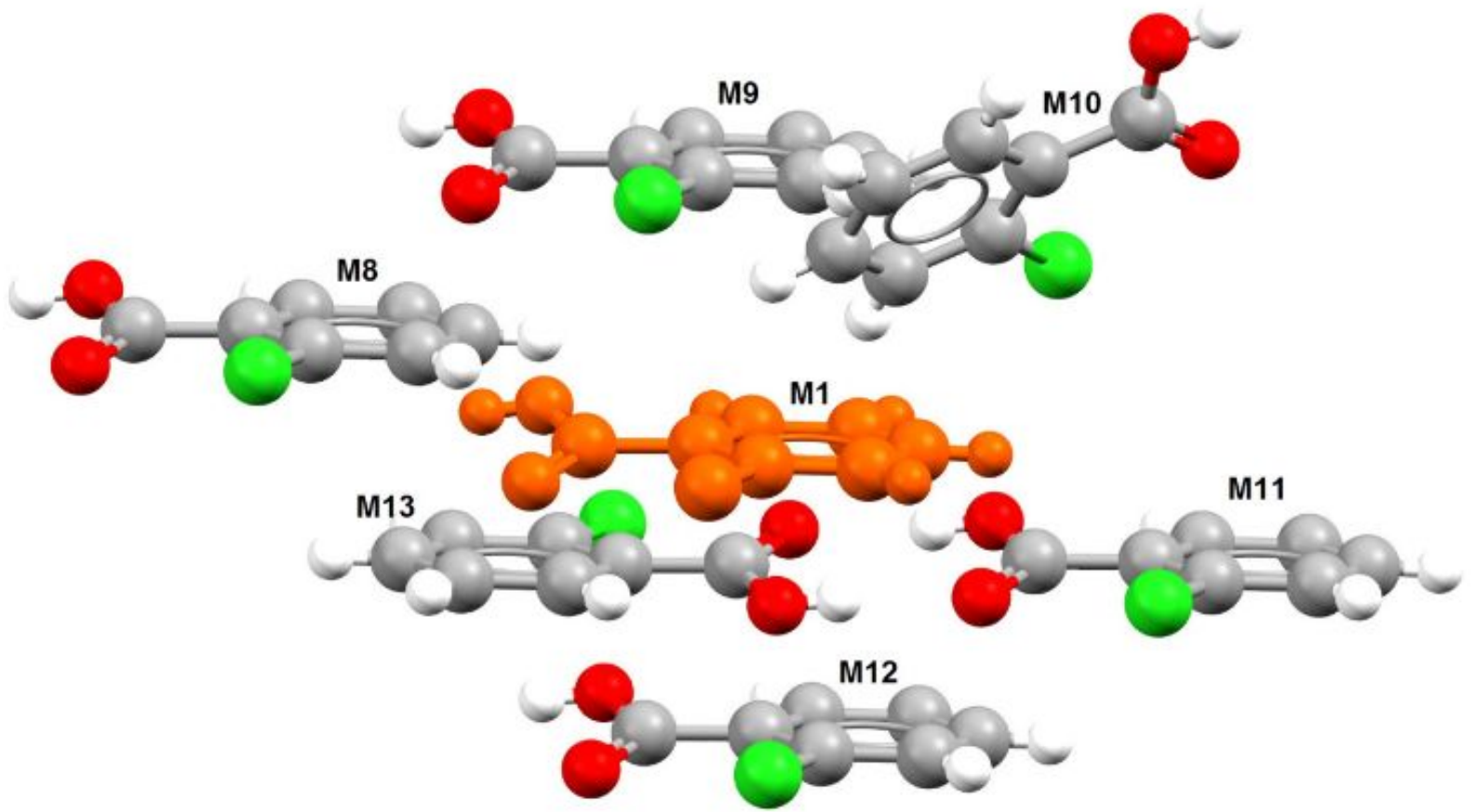

Figure S4. Supramolecular cluster showing central molecule with neighboring molecules in upper and lower layers for compound 2. 


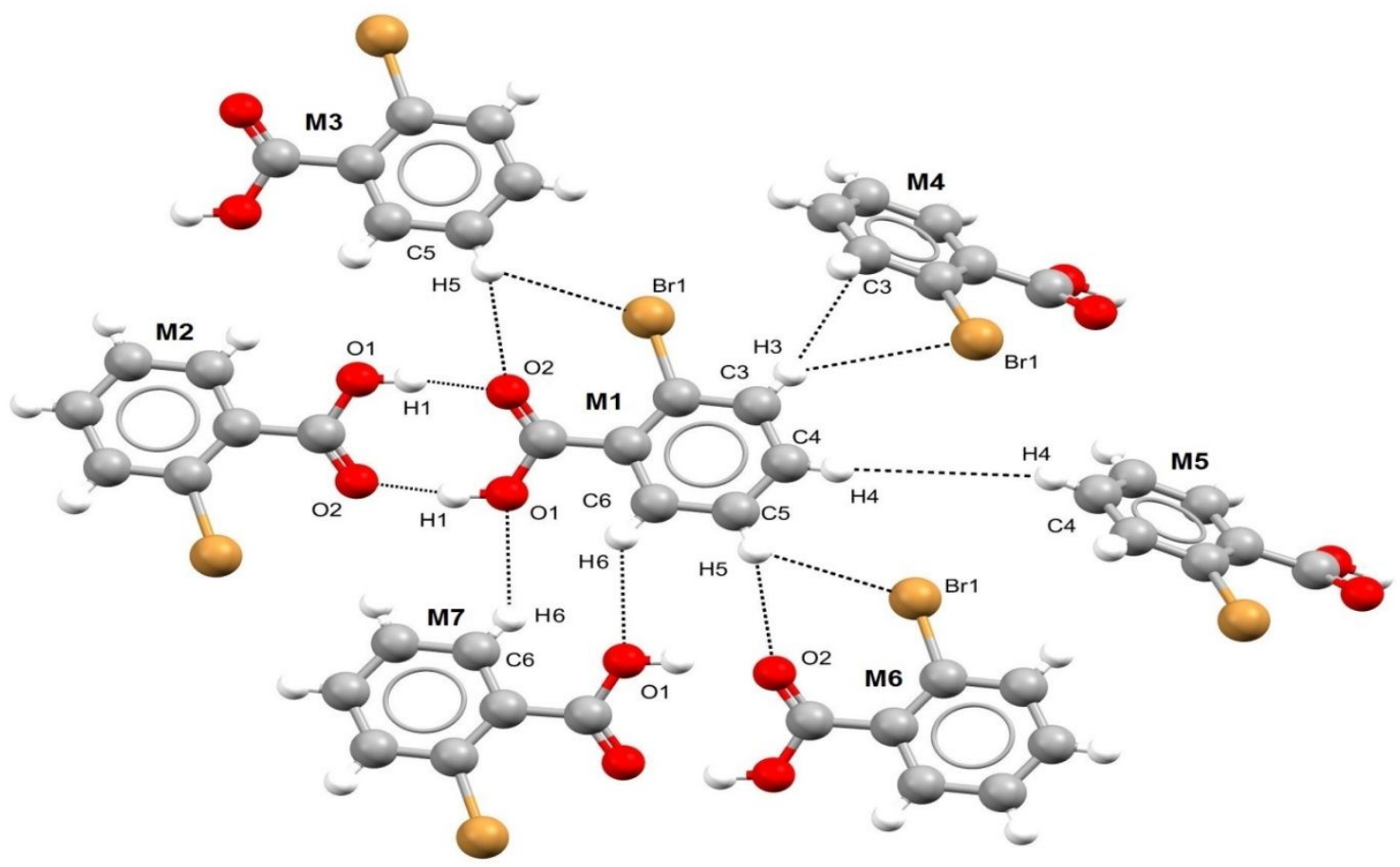

Figure S5. Supramolecular cluster showing central molecule with neighboring molecules in the same plane for compound 3.

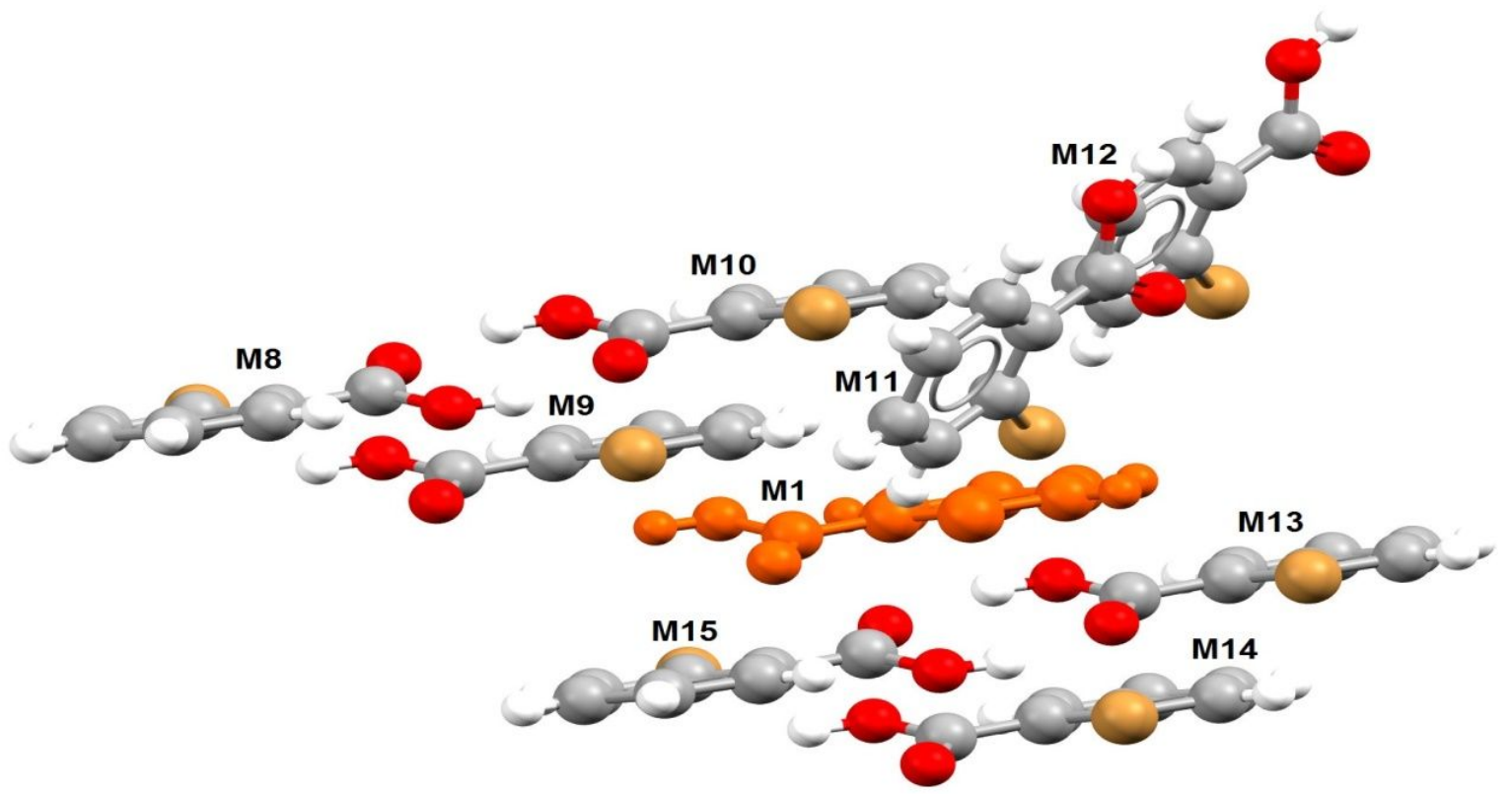

Figure S6. Supramolecular cluster showing central molecule with neighboring molecules in upper and lower layers for compound 3. 


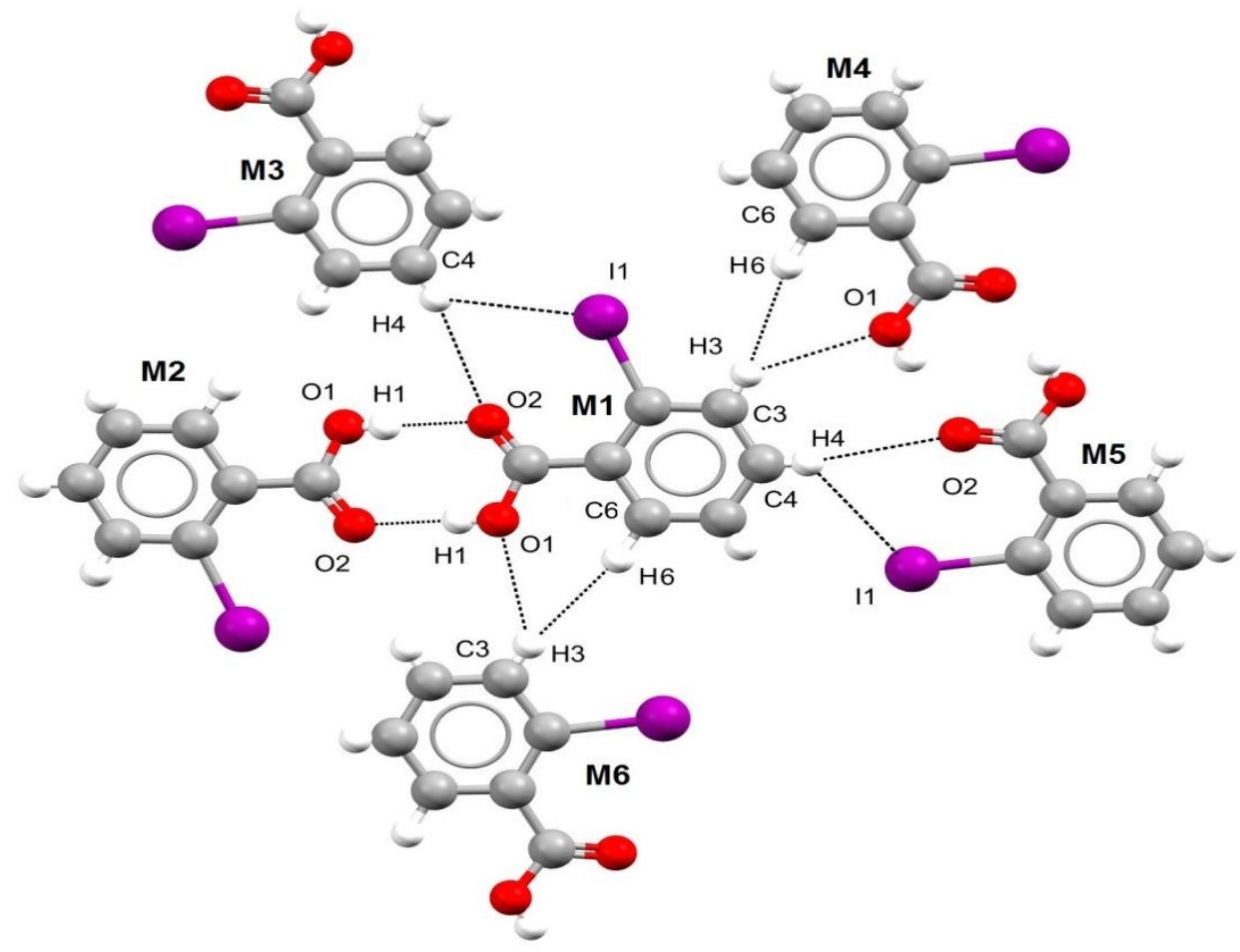

Figure S7. Supramolecular cluster showing central molecule with neighboring molecules in the same plane for compound 4.

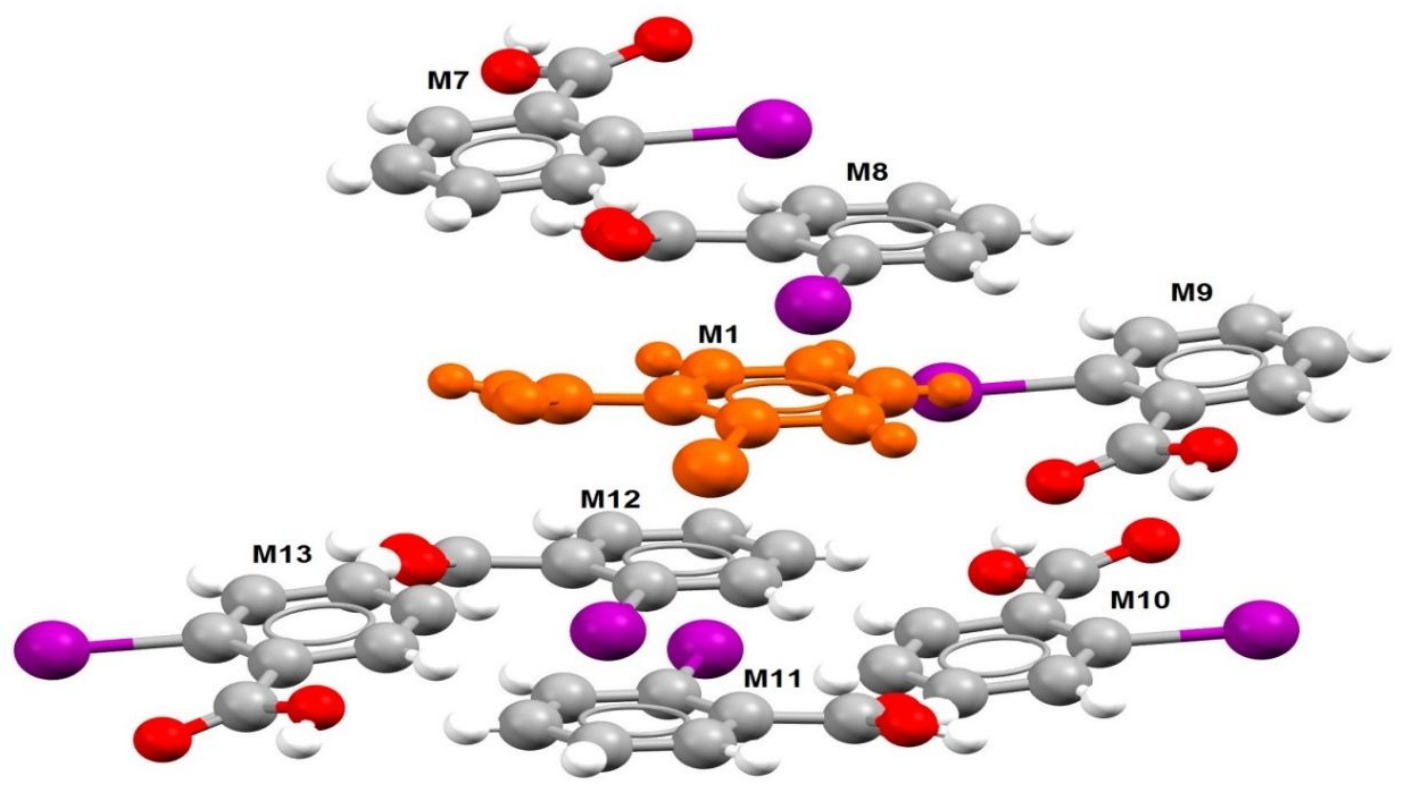

Figure S8. Supramolecular cluster showing central molecule with neighboring molecules in upper and lower layers for compound 4. 


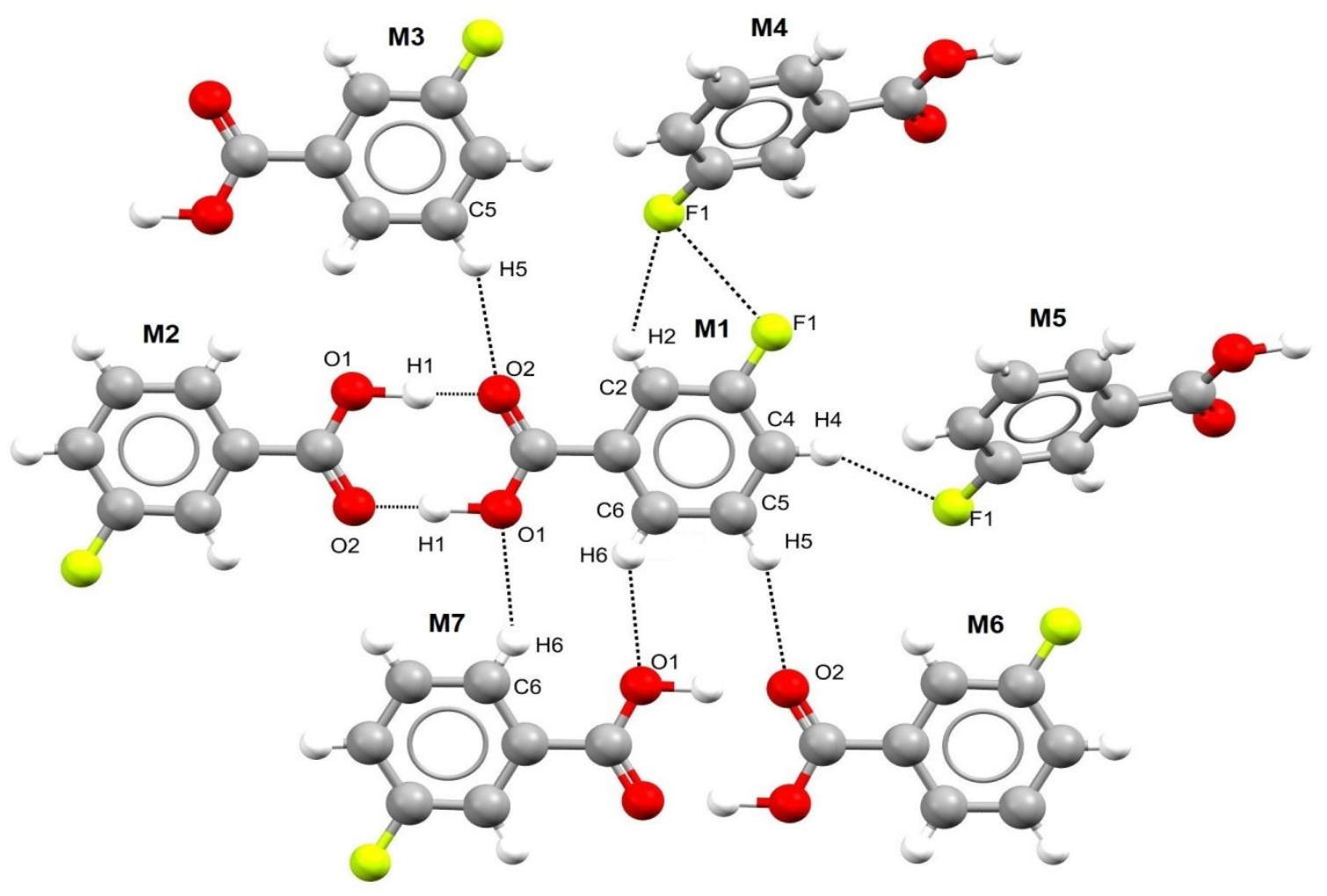

Figure S9. Supramolecular cluster showing central molecule with neighboring molecules in the same plane for compound $\mathbf{5}$.

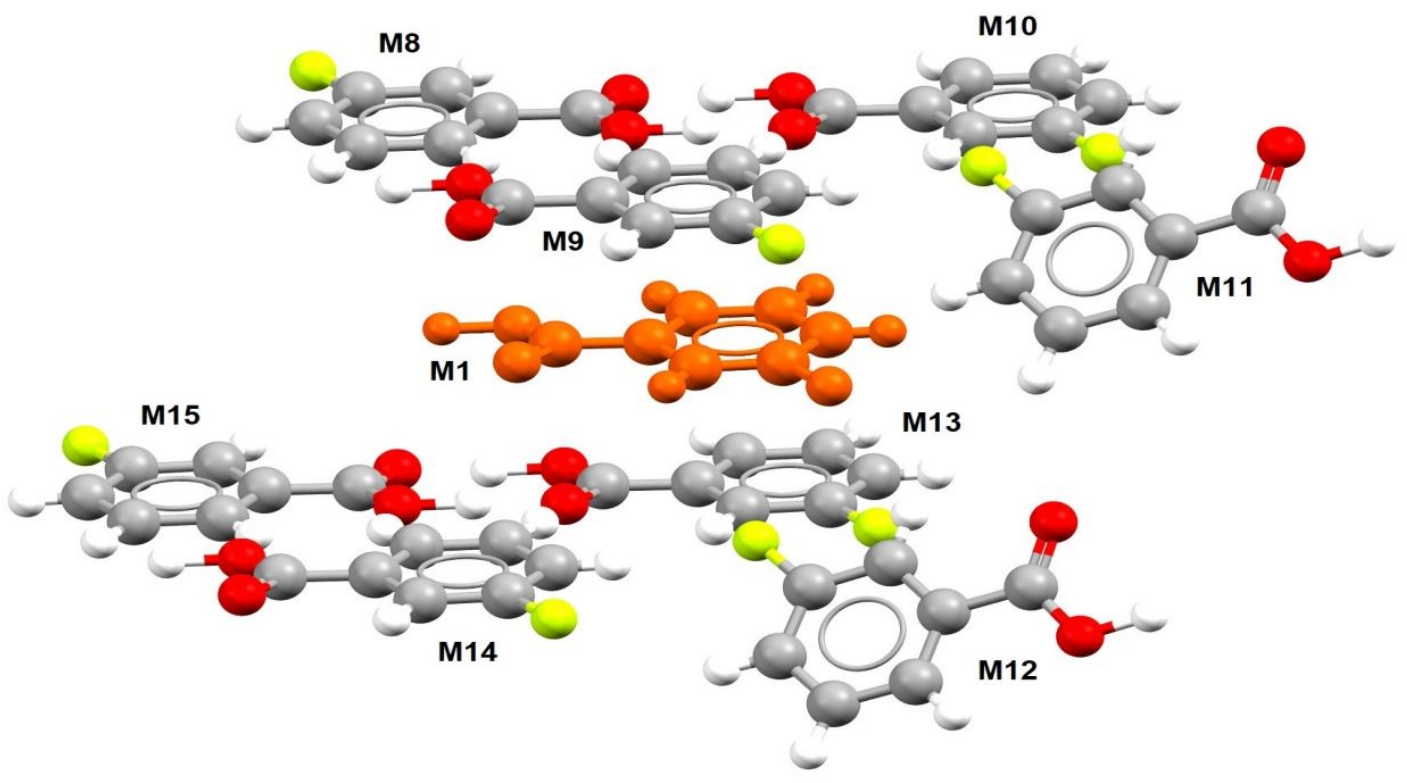

Figure S10. Supramolecular cluster showing central molecule with neighboring molecules in upper and lower layers for compound $\mathbf{5}$. 


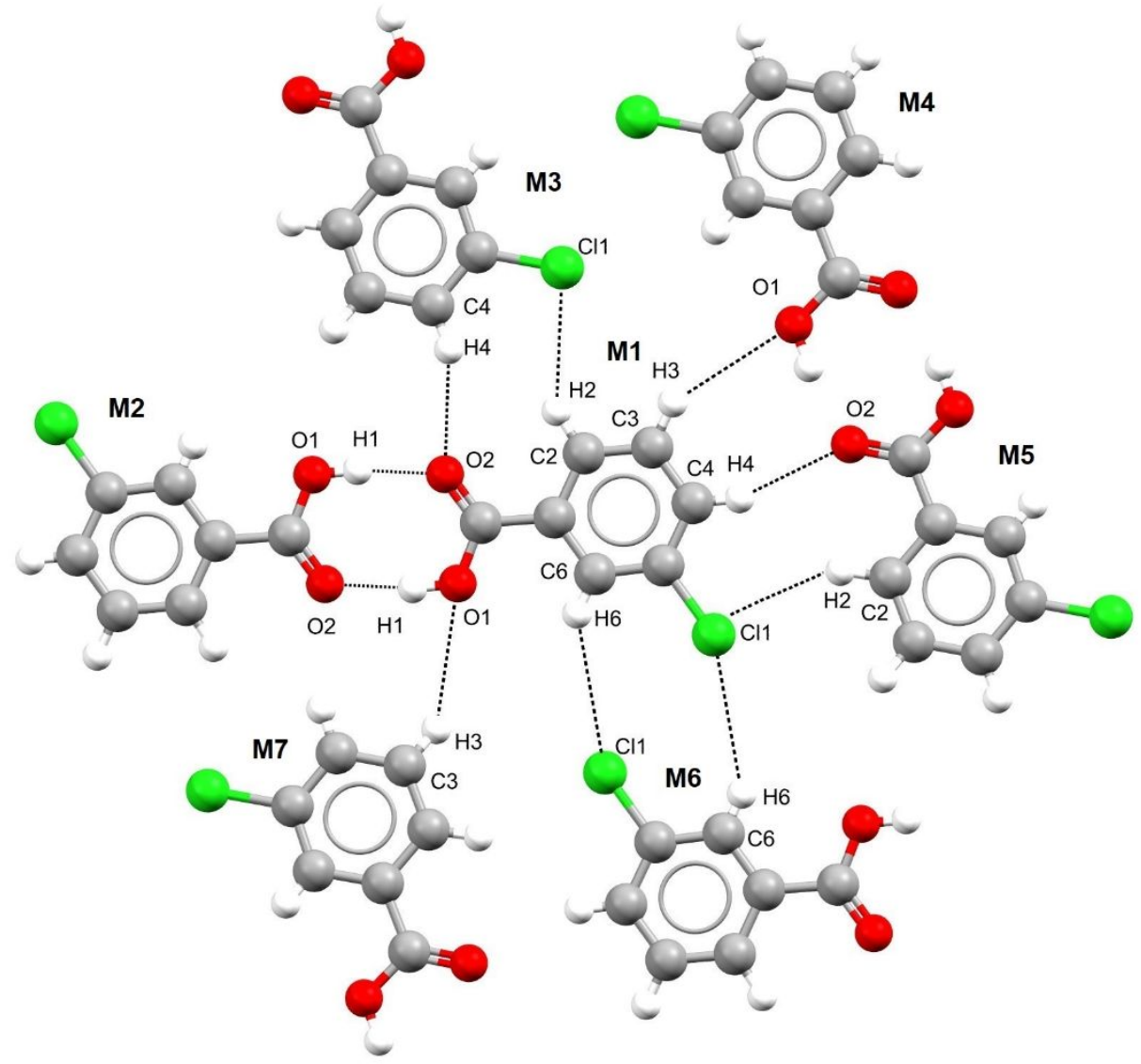

Figure S11. Supramolecular cluster showing central molecule with neighboring molecules in the same plane for compound 6.

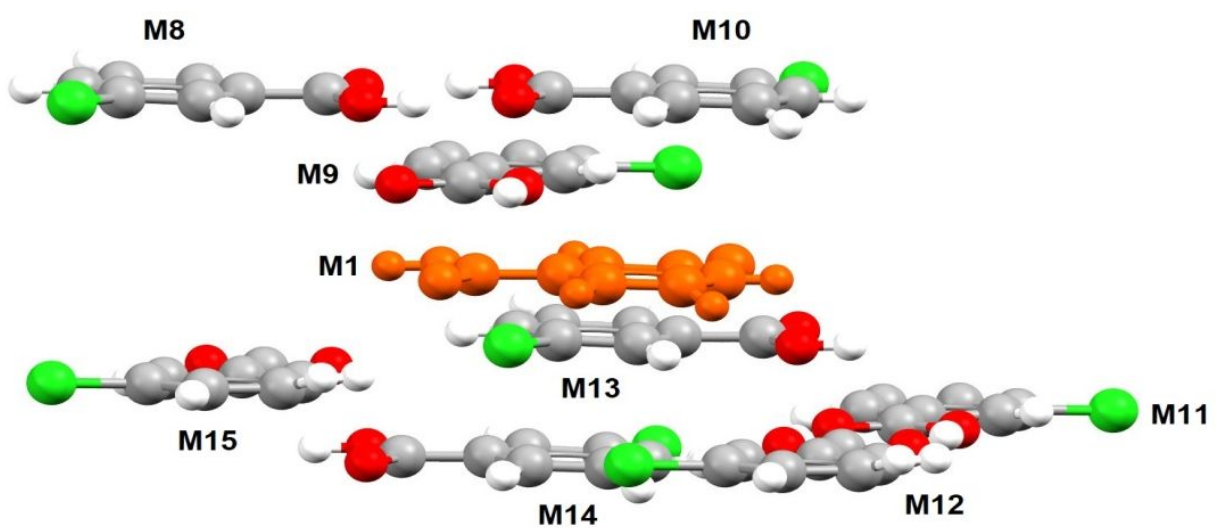

Figure S12. Supramolecular cluster showing central molecule with neighboring molecules in upper and lower layers for compound 6. 


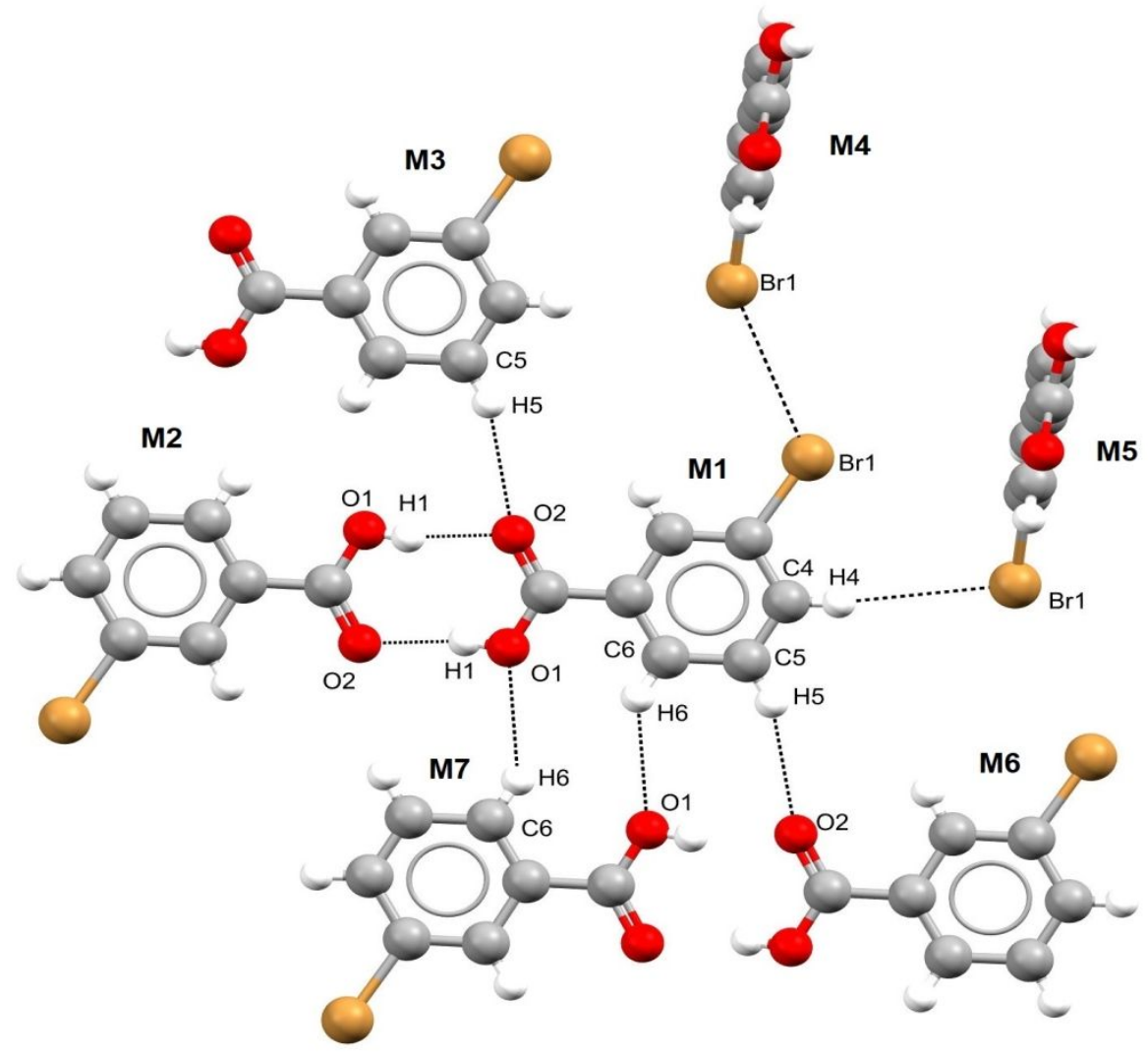

Figure S13. Supramolecular cluster showing central molecule with neighboring molecules in the same plane for compound 7.

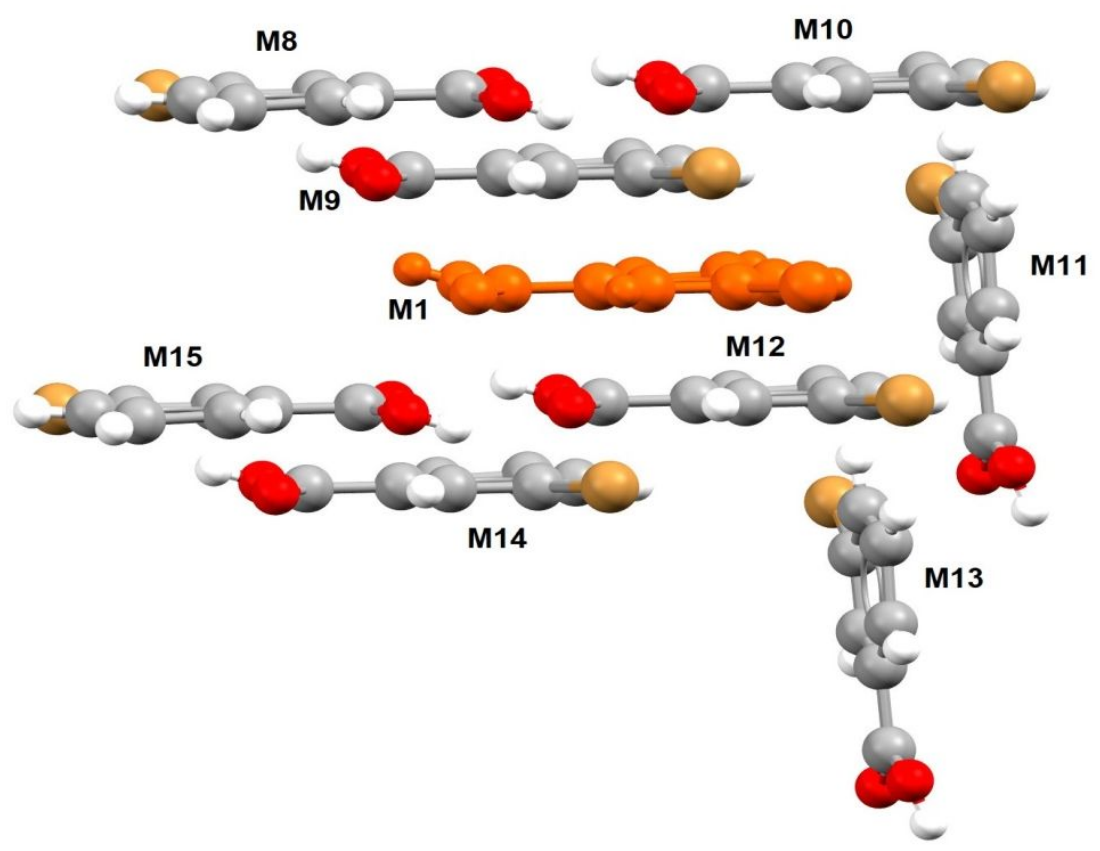

Figure S14. Supramolecular cluster showing central molecule with neighboring molecules in upper and lower layers for compound 7. 


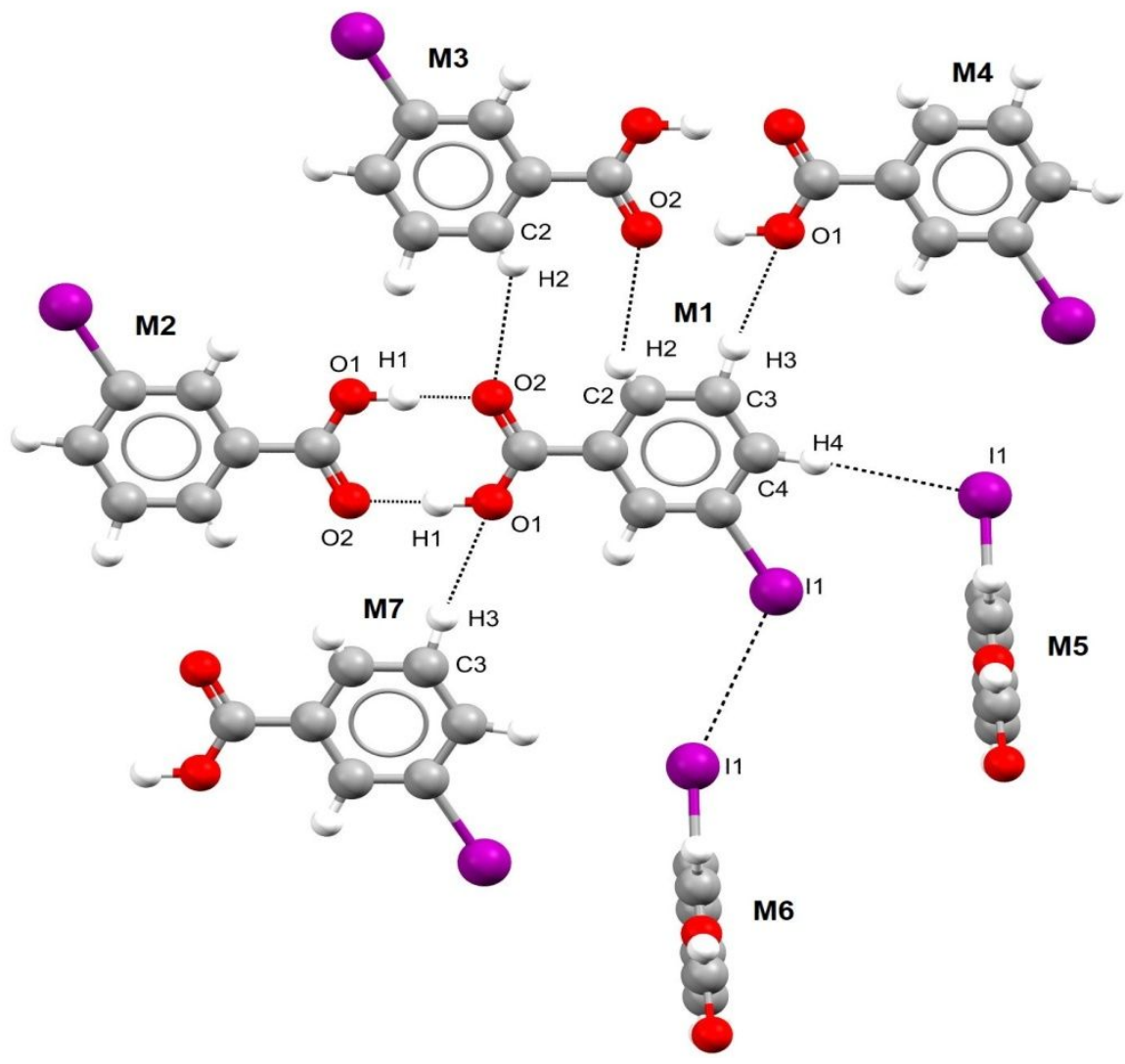

Figure S15. Supramolecular cluster showing central molecule with neighboring molecules in the same plane for compound 8.

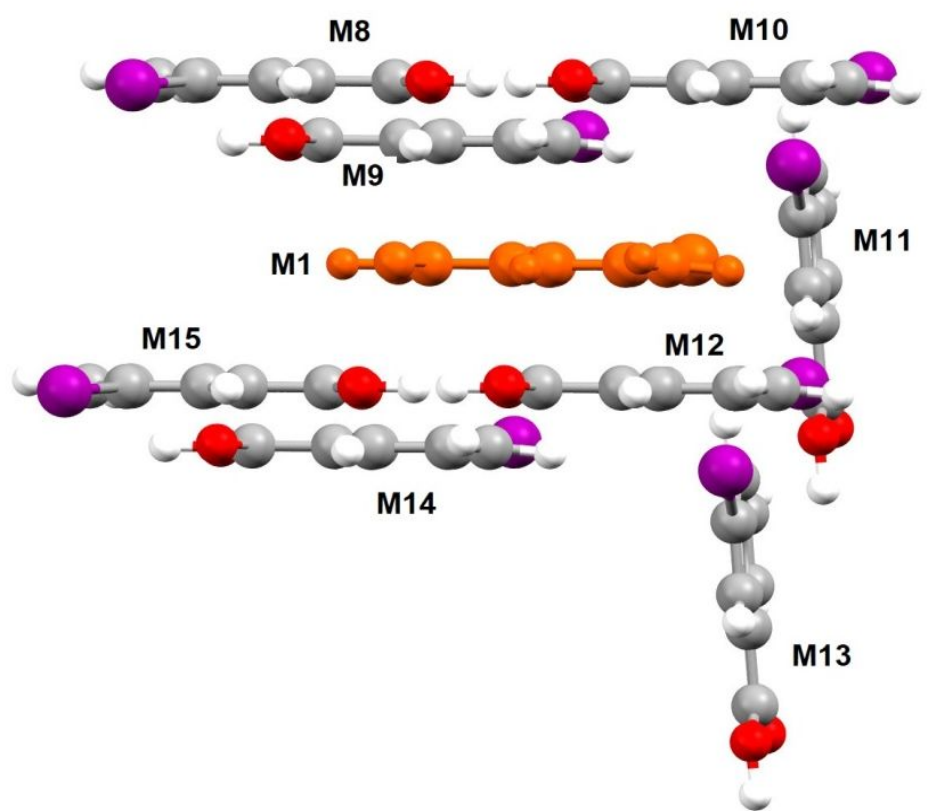

Figure S16. Supramolecular cluster showing central molecule with neighboring molecules in upper and lower layers for compound $\mathbf{8}$. 


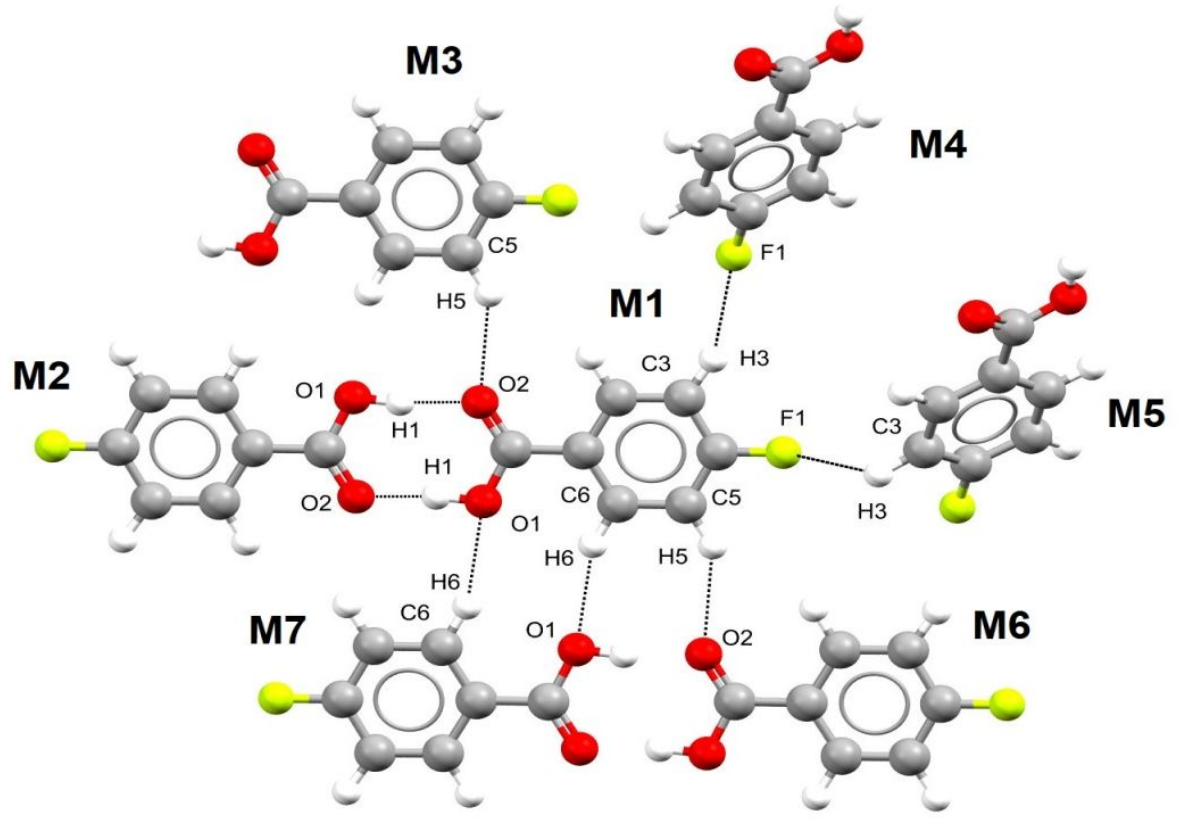

Figure S17. Supramolecular cluster showing central molecule with neighboring molecules in the same plane for compound 9.

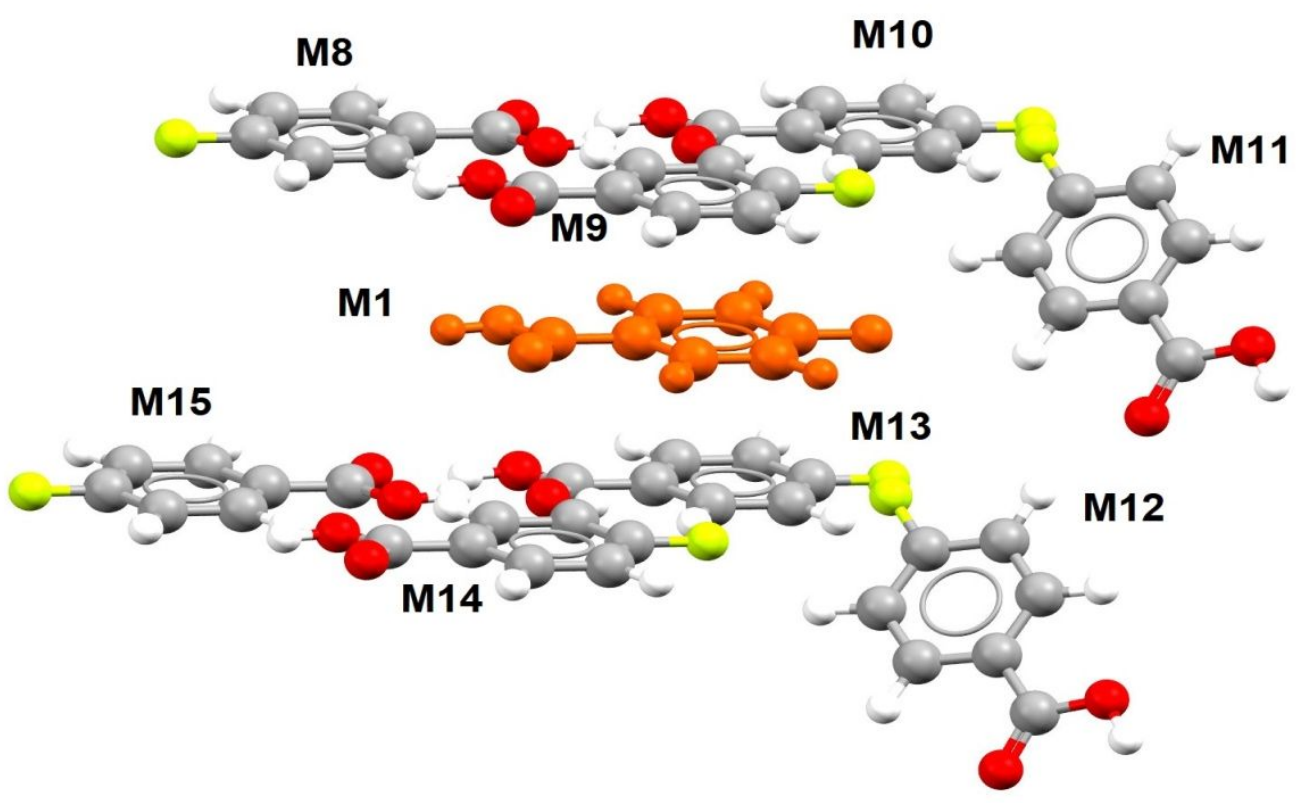

Figure S18. Supramolecular cluster showing central molecule with neighboring molecules in upper and lower layers for compound 9. 


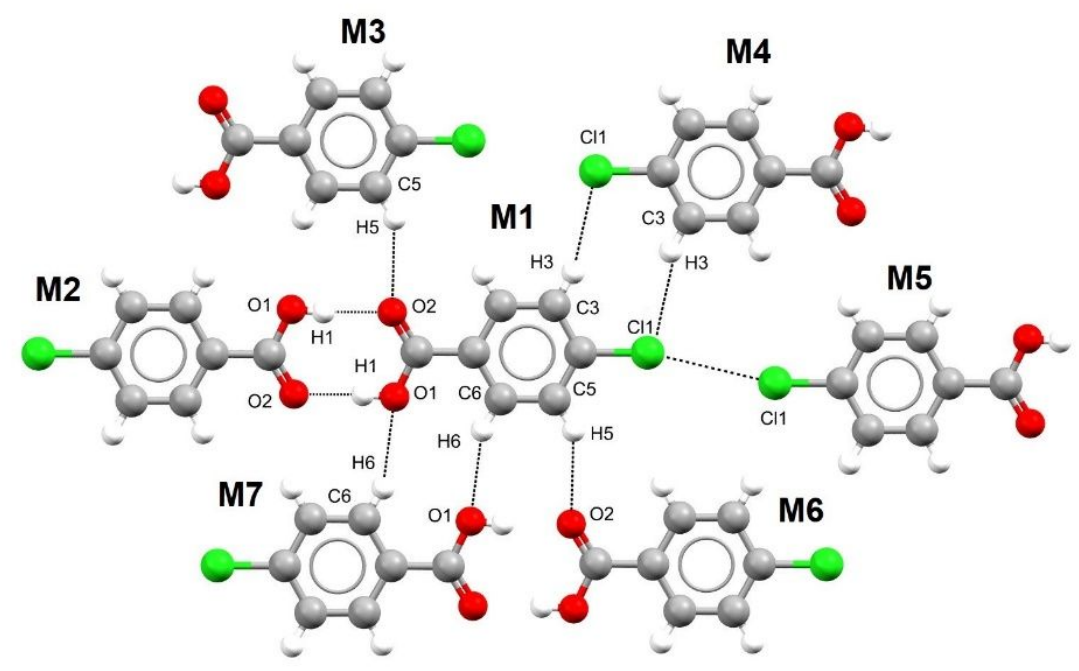

Figure S19. Supramolecular cluster showing central molecule with neighboring molecules in the same plane for compound 10.

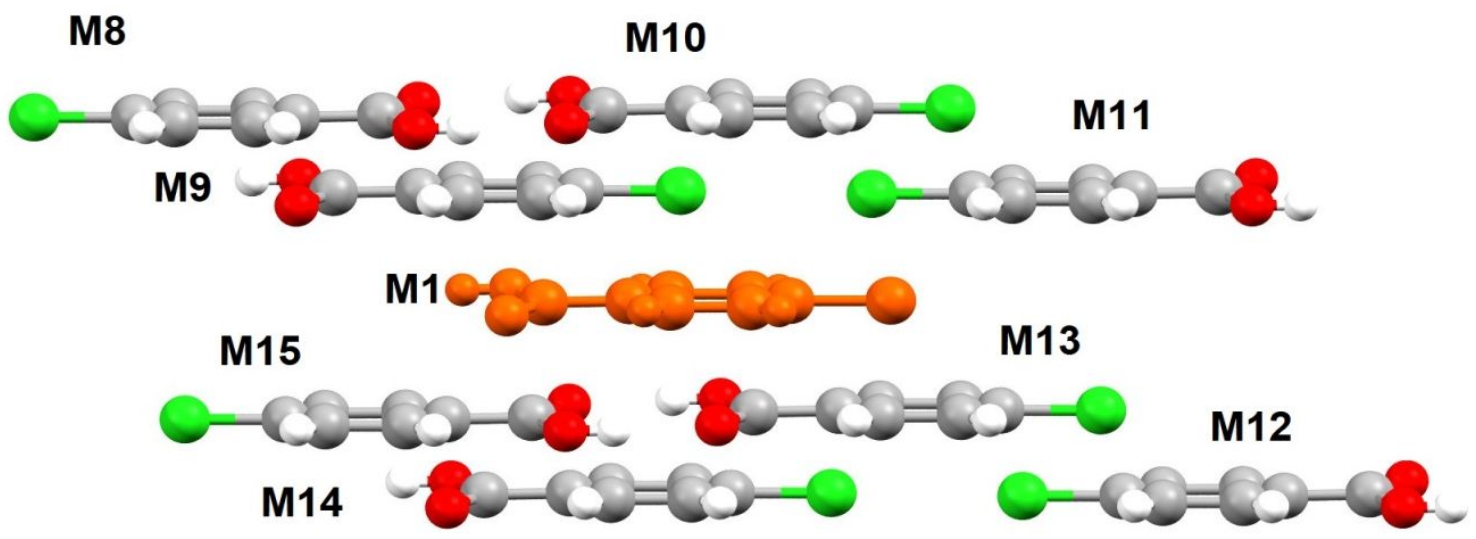

Figure S20. Supramolecular cluster showing central molecule with neighboring molecules in upper and lower layers for compound $\mathbf{1 0 .}$ 


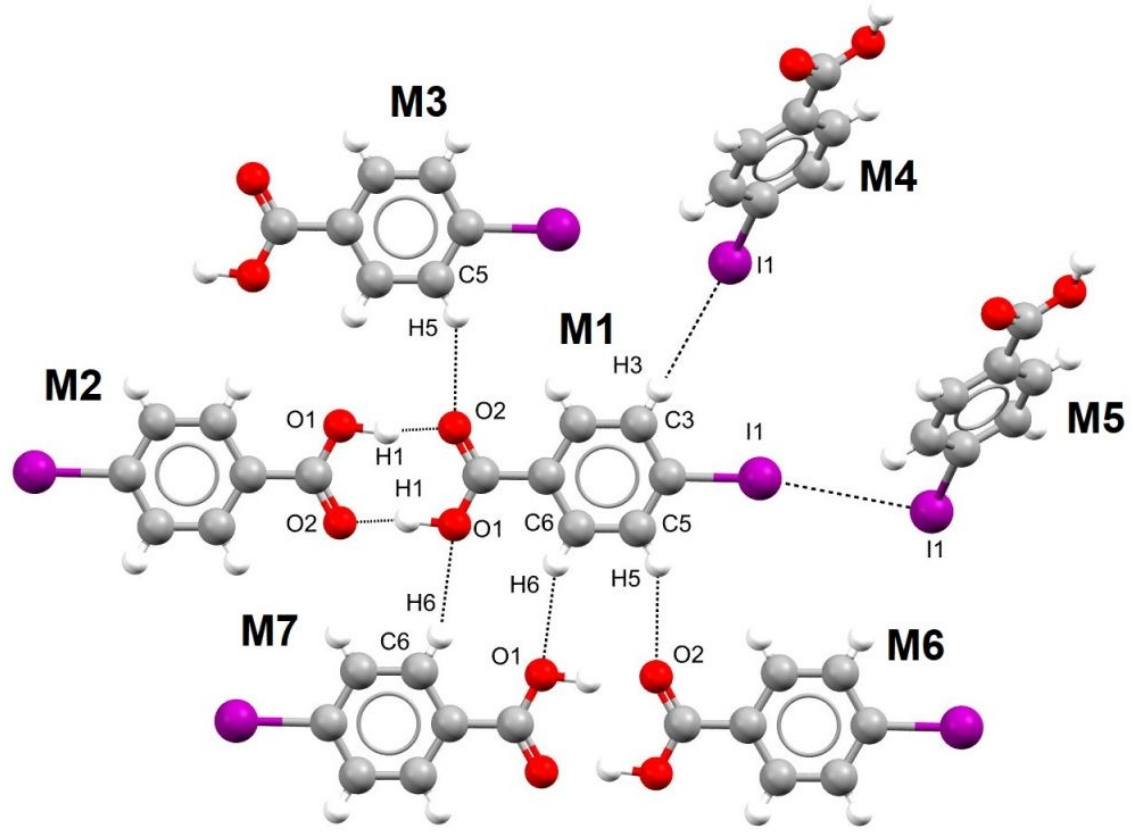

Figure S21. Supramolecular cluster showing central molecule with neighboring molecules in the same plane for compound 12.

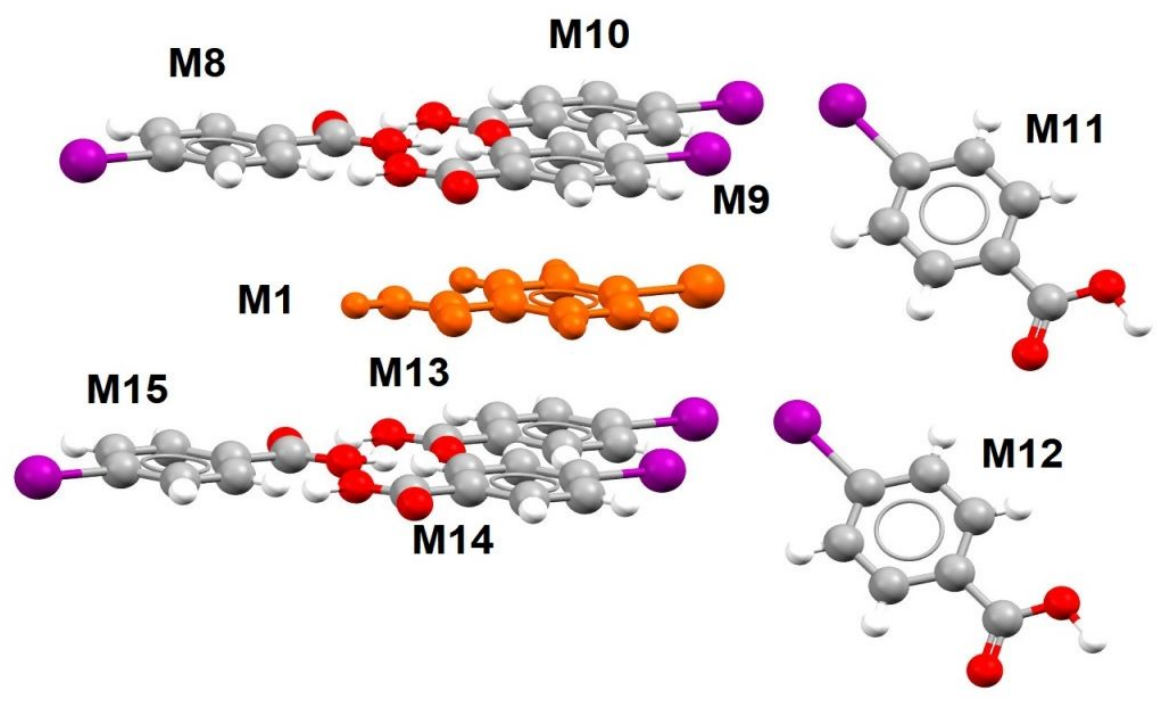

Figure S22. Supramolecular cluster showing central molecule with neighboring molecules in upper and lower layers for compound 12. 